\title{
STRUCTURES OF (SUPERSYMMETRIC) CLASSICAL W-ALGEBRAS
}

\author{
UHI RINN SUH
}

\begin{abstract}
In the first part of this paper, we discuss the classical W-algebra $\mathcal{W}(\mathfrak{g}, F)$ associated with a Lie superalgebra $\mathfrak{g}$ and the nilpotent element $F$ in an $\mathfrak{s l}_{2}$-triple. We find a generating set of $\mathcal{W}(\mathfrak{g}, F)$ and compute the Poisson brackets between them. In the second part, which is the main part of the paper, we discuss supersymmetric classical W-algebras. We introduce two different constructions of a supersymmetric classical W-algebra $\mathcal{W}(\mathfrak{g}, f)$ associated with a Lie superalgebra $\mathfrak{g}$ and an odd nilpotent element $f$ in a subalgebra isomorphic to osp $(1 \mid 2)$. The first construction is via the SUSY classical BRST complex and the second is via the SUSY DrinfeldSokolov Hamiltonian reduction. We show that these two methods give rise to isomorphic SUSY Poisson vertex algebras. As a supersymmetric analogue of the first part, we compute explicit generators and Poisson brackets between the generators.
\end{abstract}

\section{INTRODUCTION}

W-algebras were introduced by Zamolodchikov [36] and Fateev-Lukyanov [15] in the conformal field theory (see the review article by Bouwknegt-Schoutens [1] and the references therein). Drinfeld-Sokolov [13] described the classical W-algebra $\mathcal{W}\left(\mathfrak{g}_{\mathrm{ev}}\right)$ associated with a Lie algebra $\mathfrak{g}_{\mathrm{ev}}$ via a Hamiltonian reduction and related it to a hierarchy of integrable Hamiltonian systems. Feigin-Frenkel [14] constructed the (quantum) W-algebra $W\left(\mathfrak{g}_{\mathrm{ev}}\right)$ via the BRST quantization of the Drinfeld-Sokolov reduction. As a generalization, Kac-Roan-Wakimoto [23] introduced $\mathrm{W}$-algebras $W(\mathfrak{g}, F)$ associated with a finite simple Lie superalgebra $\mathfrak{g}$ and a nilpotent element $F$ in an $\mathfrak{s l}_{2}$-triple (see also [5, 24]). Note that the classical and quantum $\mathrm{W}$-algebras $\mathcal{W}\left(\mathfrak{g}_{\mathrm{ev}}\right)$ and $W\left(\mathfrak{g}_{\mathrm{ev}}\right)$, respectively, by Drinfeld-Sokolov and Feigin-Frenkel are the cases where $F$ is a principle nilpotent element.

A classical W-algebra $\mathcal{W}(\mathfrak{g}, F)$ can be understood as the classical limit of the corresponding quantum $\mathrm{W}$-algebra $W(\mathfrak{g}, F)$. More precisely, $\mathcal{W}(\mathfrak{g}, F)$ can be equivalently defined via the classical BRST complex and the generalized Drinfeld-Sokolov reduction [8, 33. The second construction shows the similarities between classical W-algebras and finite (classical) W-algebras [16, 32].

Classical W-algebras are Poisson vertex algebras (PVAs) and PVAs are the underlying algebraic structures of infinite dimensional Hamiltonian systems. Barakat-De Sole-Kac 3 investigated integrable Hamiltonian systems in terms of PVAs. In a series of papers by De SoleKac-Valeri 6, 7, 8, 9, 10, Hamiltonian integrable systems related to the classical W-algebras $\mathcal{W}\left(\mathfrak{g}_{\mathrm{ev}}, F\right)$ were constructed. Moreover, they computed explicit forms of generators of $\mathcal{W}\left(\mathfrak{g}_{\mathrm{ev}}, F\right)$ and Poisson brackets between the the generators [11.

The author investigated in 34] the algebraic properties of classical W-algebras $\mathcal{W}(\mathfrak{g}, F)$ associated with Lie superalgebras $\mathfrak{g}$. The following properties are crucially used in this paper:

- As a differential algebra, $\mathcal{W}(\mathfrak{g}, F) \simeq S(\mathbb{C}[\partial] \otimes \operatorname{ker} F)$, where $\partial$ is the even derivation in the supersymmetric algebra $S(\mathbb{C}[\partial] \otimes \operatorname{ker} F)$. 
- $\mathcal{W}(\mathfrak{g}, F)=\mathbb{C}\left[\partial^{n} J_{i} \mid n \in \mathbb{Z}_{\geq 0}, i \in I\right]$ for $J_{i}=u_{i}+A_{i}$ where $\left\{u_{i} \mid i \in I\right\}$ is a basis of ker ad $F$ and $A_{i}$ consists of total derivative parts and degree $\geq 2$ parts.

Using the above mentioned properties, explicit forms of generators and Poisson brackets between them are presented in Section 3. In addition, a partial result on related Hamiltonian integrable systems has been written [35].

The supersymmetric counterpart of the W-algebras, the so-called supersymmetric (SUSY) W-algebras were introduced by Madsen-Ragoucy [27] via SUSY BRST complexes. A SUSY Walgebra $W(\mathfrak{g}, f)$ is constructed for simple Lie superalgebra $\mathfrak{g}$ and odd nilpotent $f$ in a subalgebra isomorphic to $\mathfrak{o s p}(1 \mid 2)$. In the article by Heluani-Kac [17, they provided the notion and basic properties of SUSY vertex algebras (see also [22]). Molev, Ragoucy and the author 28] showed that the SUSY BRST complexes and SUSY W-algebras can be described within SUSY vertex algebra theory.

SUSY classical W-algebras, the most crucial ingredients of this paper, have been studied as Poisson structures of super integrable systems [4, 12, 18, 19, 20, 21, 25, 26, 29, 30, 31. In particular, in various articles such as [4, 12, 19, 18, super integrable systems associated with Lie superalgebras have been constructed and the related Poisson algebras are called SUSY Walgebras associated with Lie superalgebras. These arguments can be viewed as supersymmetric generalizations of Drinfeld-Sokolov reductions and hierarchies.

The first part of this paper (Sections 2 and 3) deals with (nonSUSY) classical W-algebras $\mathcal{W}(\mathfrak{g}, F)$. In Section 2, we review the basic properties of PVAs and classical W-algebras. In particular, the crucial facts used in Section 3 are the master formula for Poisson $\lambda$-brackets (see Proposition 2.5 ) and the properties of free generators of $\mathcal{W}(\mathfrak{g}, F)$ (see Proposition 2.10). Using these propositions, we find generators of $\mathcal{W}(\mathfrak{g}, F)$ and Poisson $\lambda$-brackets between the generators. These results are analogous to the results for classical $\mathrm{W}$-algebras associated with Lie algebras in [11].

The second part (Section 4, 5, 6) is the supersymmetric analogue of the first part. Accordingly, we review the basics of SUSY Poisson vertex algebras in Section 4. In Section 5, we clarify the definition of a SUSY classical W-algebra $\mathcal{W}(\mathfrak{g}, f)$. There are two natural ways to construct $\mathcal{W}(\mathfrak{g}, f)$ :

(i) via SUSY classical BRST complexes (Theorem [5.6)

(ii) using SUSY Drinfeld-Sokolov Hamiltonian reductions (Definition 5.2)

In Theorem 5.13, we prove that (i) and (ii) are isomorphic as SUSY PVAs. The SUSY classical BRST complexes can be obtained by the classical limit of SUSY BRST complexes [28]. The classical limit of SUSY vertex algebras has been studied by Heluani-Kac [17]. As for quantum and classical W-algebras and SUSY quantum W-algebras [23, 24, 5, 34, 28], BRST complexes allow us to find the number of free generators of $\mathcal{W}(\mathfrak{g}, f)$ and the following properties of generators (see Proposition 5.12):

- As a differential algebra, $\mathcal{W}(\mathfrak{g}, f) \simeq S(\mathbb{C}[D] \otimes \operatorname{ker} \operatorname{ad} f)$, where $D$ is the odd derivation.

- $\mathcal{W}(\mathfrak{g}, f)=\mathbb{C}\left[D^{n} J_{i} \mid n \in \mathbb{Z}_{>0}, i \in I_{S}\right]$ for $J_{i}=u_{i}+A_{i}$, where $\left\{u_{i} \mid i \in I_{S}\right\}$ is a basis of ker ad $f$ and $A_{i}$ consists of total derivative parts and degree $\geq 2$ parts.

Furthermore, using the second definition (ii) of $\mathcal{W}(\mathfrak{g}, f)$, we find its algebraic structures analogous to those of nonSUSY classical W-algebras. More precisely, we can find the explicit forms of elements in $\mathcal{W}(\mathfrak{g}, f)$ by finding $J_{i}$ 's (Theorem 6.3). For the Poisson $\chi$-brackets between generators (Theorem 6.5), we use the SUSY master formula [4]. We plan to study relations between 
the nonSUSY classical W-algebra $\mathcal{W}\left(\mathfrak{g}, F=f^{2}\right)$ and the SUSY classical W-algebra $\mathcal{W}(\mathfrak{g}, f)$ with Victor Kac, as part of our future work.

Acknowledgments The author was supported by the New Faculty Startup Fund from Seoul National University and the NRF Grant \# NRF-2019R1F1A1059363.

\section{Poisson vertex algebras and Classical W-algebras}

In this section, we recall notions related to Poisson vertex algebras introduced in [2] and classical W-algebras. Note that, throughout this paper, the base field is $\mathbb{C}$.

2.1. Poisson vertex algebras. A $\mathbb{Z} / 2 \mathbb{Z}$-graded vector space $V=V_{\overline{0}} \oplus V_{\overline{1}}$ is called a vector superspace and a homogeneous element $a \in V_{\bar{i}}$ is called an even (resp. odd) element if $i=0$ (resp. $i=1$ ). Denote $p(a):=i, s(a)=(-1)^{i}$ and $s(a, b)=(-1)^{i j}$ for $a \in V_{\bar{i}}$ and $b \in V_{\bar{j}}$. An operator $\phi: V \rightarrow V$ is called even if $\phi\left(V_{\bar{i}}\right) \subset V_{\bar{i}}$ and odd if $\phi\left(V_{\bar{i}}\right) \subset V_{\bar{i}+\overline{1}}$. A $\mathbb{C}$-algebra $A$ is called a superalgebra if $A$ is a vector superspace such that $A_{\bar{i}} A_{\bar{j}} \subset A_{\bar{i}+\bar{j}}$.

Let $R=R_{\overline{0}} \oplus R_{\overline{1}}$ be a $\mathbb{C}[\partial]$-module with even operator $\partial: R \rightarrow R$. Consider the even indeterminate $\lambda$ and $\mathbb{C}[\partial]$-module $\mathbb{C}[\lambda] \otimes R$ via

$$
\partial\left(\lambda^{n} \otimes a\right)=\lambda^{n} \otimes \partial a
$$

for $a \in R$. Note that we usually omit $\otimes$ in elements of $\mathbb{C}[\lambda] \otimes R$.

Definition 2.1. Suppose the $\mathbb{C}[\partial]$-module $R$ endowed with the linear $\lambda$-bracket

$$
\left[{ }_{\lambda}\right]: R \otimes_{\mathbb{C}} R \rightarrow \mathbb{C}[\lambda] \otimes R
$$

satisfies the following properties:

- (parity preserving) $p(a)+p(b)=p\left(\left[a_{\lambda} b\right]\right)$,

- (sesquilinearity) $\left[a_{\lambda} \partial b\right]=(\partial+\lambda)\left[a_{\lambda} b\right]$ and $\left[\partial a_{\lambda} b\right]=-\lambda\left[a_{\lambda} b\right]$,

- (skew-symmetry) $\left[a_{\lambda} b\right]=-s(a, b)\left[b_{-\lambda-\partial} a\right]$,

- $\left(\right.$ Jacobi identity) $\left[a_{\lambda}\left[b_{\mu} c\right]\right]=\left[\left[a_{\lambda} b\right]_{\lambda+\mu} c\right]+s(a, b)\left[b_{\mu}\left[a_{\lambda} c\right]\right]$ in $\mathbb{C}[\lambda, \mu] \otimes R$,

for $a, b, c \in R$. Then $R$ is called a Lie conformal algebra (LCA).

To be precise, if $R$ is a LCA and we write

$$
\left[a_{\lambda} b\right]=\sum_{n \in \mathbb{Z}_{\geq 0}} \lambda^{n} a_{(n)} b
$$

for $a, b, a_{(n)} b \in R$, terms in the skew-symmetry and Jacobi identity are

- $\left[b_{-\lambda-\partial} a\right]=\sum_{n \in \mathbb{Z}_{\geq 0}}(-\lambda-\partial)^{n} a_{(n)} b$,

- $\left[a_{\lambda}\left[b_{\mu} c\right]\right]=\sum_{n, m \in \mathbb{Z}_{\geq 0}} \lambda^{n} \mu^{m} a_{(n)}\left(b_{(m)} c\right),\left[b_{\mu}\left[a_{\lambda} c\right]\right]=\sum_{n, m \in \mathbb{Z}_{\geq 0}} \mu^{n} \lambda^{m} b_{(n)}\left(a_{(m)} c\right)$,

- $\left[\left[a_{\lambda} b\right]_{\lambda+\mu} c\right]=\sum_{n, m \in \mathbb{Z}_{\geq 0}} \lambda^{n}(\mu+\lambda)^{m}\left(a_{(n)} b\right)_{(m)} c$,

where $\lambda \mu=\mu \lambda$ and $\mu \partial=\partial \mu$.

Remark 2.2. In other articles such as [5, 11, 34, 35, the coefficient of $\lambda^{n}$ in $\left[a_{\lambda} b\right]$ is denoted by $\frac{1}{n !} a_{(n)} b$. However, for simplicity of notation, we denote the coefficient by $a_{(n)} b$ as in (2.1).

Definition 2.3. A quadruple $\left(P, \partial,\left\{_{\lambda}\right\}, \cdot\right)$ is a Poisson vertex algebra $(P V A)$ if it satisfies

- $(P, \partial, \cdot)$ is a unital super-commutative differential algebra with even derivation $\partial: P \rightarrow$ $P$, i.e., $\partial(a b)=\partial(a) b+a \partial(b)$ for $a, b \in P$,

- $(P, \partial,\{\lambda\})$ is an LCA,

- (Leibniz rule) $\left\{a_{\lambda} b c\right\}=s(a, b) b\left\{a_{\lambda} c\right\}+\left\{a_{\lambda} b\right\} c$. 
Note that the Leibniz rule in Definition 2.3 is also called the right Leibniz rule. By the skew-symmetry and right Leibniz rule of LCAs, the left Leibniz rule

$$
\left\{a b_{\lambda} c\right\}=s(b, c)\left\{a_{\lambda+\partial} c\right\}_{\rightarrow} b+s(a, b c)\left\{b_{\lambda+\partial} c\right\}_{\rightarrow} a
$$

holds. Here,

$$
\left\{a_{\lambda+\partial} c\right\}_{\rightarrow} b=\sum_{n \in \mathbb{Z}_{\geq 0}} a_{(n)} c(\lambda+\partial)^{n} b .
$$

As in Definition 2.3, a PVA is a LCA. On the other hand, for a given LCA, one can generate a PVA in the following manner. We refer to [3, 35] for the following propositions (Proposition 2.4 and Proposition 2.5).

\section{Proposition 2.4.}

(1) Let $R$ be a LCA. Then the supersymmetric algebra $S(R)$ generated by $R$ is a PVA endowed with the $\lambda$-bracket defined by the $\lambda$-bracket on $R$ and the Leibniz rule.

(2) Let $V$ be a vector superspace and $P=S(\mathbb{C}[\partial] \otimes V)$, the supersymmetric algebra generated by $\bigoplus_{n \in \mathbb{Z}_{>0}} \partial^{n} V$. If a bracket $\left[{ }_{\lambda}\right]: V \otimes V \rightarrow \mathbb{C}[\lambda] \otimes P$ satisfies the skew-symmetry and Jacobi identity then it can be uniquely extended to a PVA bracket on $P$ via the sesquilinearity of LCAs and the Leibniz rule of PVAs.

Let $V=V_{\overline{0}} \oplus V_{\overline{1}}$ be a vector superspace and let $\mathcal{B}=\mathcal{B}_{\overline{0}} \cup \mathcal{B}_{\overline{1}}$ be a basis of $V$ such that $\mathcal{B}_{\overline{0}}:=\left\{u_{i} \mid i \in I_{\overline{0}}\right\}$ and $\mathcal{B}_{\overline{1}}:=\left\{u_{i} \mid i \in I_{\overline{1}}\right\}$ are bases of $V_{\overline{0}}$ and $V_{\overline{1}}$, respectively. Consider the supersymmetric differential algebra $P=S(\mathbb{C}[\partial] \otimes V)$ generated by $V$ and denote $u_{i}^{(m)}:=\partial^{m} u_{i}$ for $m \in \mathbb{Z}_{\geq 0}$. Then, as an algebra of polynomials, $P=\mathbb{C}\left[u_{i}^{(m)} \mid i \in I_{\overline{0}} \cup I_{\overline{1}}, m \in \mathbb{Z}_{\geq 0}\right]$.

For $u_{i} \in \mathcal{B}$, define the derivation $\frac{\partial}{\partial u_{i}^{(m)}}$ on $P$ of parity $p\left(u_{i}\right)$ via

$$
\frac{\partial}{\partial u_{i}^{(m)}} u_{j}^{(n)}=\delta_{m, n} \delta_{i, j}, \quad \frac{\partial}{\partial u_{i}^{(m)}}(f g)=\frac{\partial}{\partial u_{i}^{(m)}}(f) g+s\left(u_{i}, f\right) f \frac{\partial}{\partial u_{i}^{(m)}}(g) .
$$

Proposition 2.5 (Master formula for PVA). If the differential supersymmetric algebra $P$ is a $P V A$ endowed with the bracket $\left\{_{\lambda}\right\}$ then

$$
\left\{f_{\lambda} g\right\}=\sum_{\substack{i, j \in I \\ m, n \in \mathbb{Z} \geq 0}} C_{i, j}^{f, g} \frac{\partial g}{\partial u_{j}^{(n)}}(\lambda+\partial)^{n}\left\{u_{i \lambda+\partial} u_{j}\right\}_{\rightarrow}(-\lambda-\partial)^{m} \frac{\partial f}{\partial u_{i}^{(m)}},
$$

where $C_{i, j}^{f, g}:=s(f, g) s\left(u_{i}, u_{j}\right) s\left(g, u_{i}\right) s\left(u_{i}\right)$ for $f, g \in P$.

Example 2.6. Let $\mathfrak{g}$ be a Lie superalgebra with an invariant bilinear form $(\mid)$. The supersymmetric differential algebra $P=S(\mathbb{C}[\partial] \otimes \mathfrak{g})$ generated by $\mathfrak{g}$ is called the affine PVA if it is endowed with the bracket defined by

$$
\left\{a_{\lambda} b\right\}=[a, b]+k \lambda(a \mid b), \quad a, b \in \mathfrak{g}
$$

for $k \in \mathbb{C}$. By Proposition 2.4. (2.3) completely determines the PVA structure of $P$ and, by Proposition 2.5, the $\lambda$-bracket of any two elements can be computed directly.

2.2. Classical $\mathbf{W}$-algebras. Let $\mathfrak{g}$ be a finite simple Lie superalgebra with an $\operatorname{sl}_{2}$-triple $(E, H, F)$ and the nondegenerate even supersymmetric invariant bilinear form $(\mid)$ such that $(E \mid F)=$ $\frac{1}{2}(H \mid H)=1$. Then

$$
\mathfrak{g}=\bigoplus_{i \in \frac{\mathbb{Z}}{2}} \mathfrak{g}(i)
$$


is the eigenspace decomposition with respect to ad $\frac{H}{2}$. Let us write

$$
\mathfrak{g}_{\geq j}=\bigoplus_{i \geq j} \mathfrak{g}(i), \quad \mathfrak{g}_{\leq j}=\bigoplus_{i \leq j} \mathfrak{g}(i), \quad \mathfrak{g}_{>j}=\bigoplus_{i>j} \mathfrak{g}(i), \quad \mathfrak{g}_{<j}=\bigoplus_{i<j} \mathfrak{g}(i) .
$$

The super Lie subalgebras $\mathfrak{n}$ and $\mathfrak{m}$ and the subspace $\mathfrak{p}$ of $\mathfrak{g}$ are defined by

$$
\mathfrak{n}=\mathfrak{g}_{>0}, \quad \mathfrak{m}=\mathfrak{g}_{\geq 1}, \quad \mathfrak{p}=\mathfrak{g}_{<1} .
$$

Consider the differential superalgebra $P(\mathfrak{g})=S(\mathbb{C}[\partial] \otimes \mathfrak{g})$ and its ideal $\mathcal{I}_{F}$ generated by $m-(F \mid m)$ for $m \in \mathfrak{m}$. Recall that $P(\mathfrak{g})$ endowed with the $\lambda$-bracket (2.3) is called the affine PVA. Let $\operatorname{ad}_{\lambda} n: P(\mathfrak{g}) \rightarrow \mathbb{C}[\lambda] \otimes P(\mathfrak{g})$ for $n \in \mathfrak{n}$ be defined by:

$$
\operatorname{ad}_{\lambda} n(A):=\left\{n_{\lambda} A\right\} \text { for } n \in \mathfrak{n}, A \in P(\mathfrak{g}) .
$$

Since $\operatorname{ad}_{\lambda} n\left(\mathcal{I}_{F}\right) \subset \mathbb{C}[\lambda] \otimes \mathcal{I}_{F}$, (2.7) canonically induces the function from $P(\mathfrak{g}) / \mathcal{I}_{F}$ to $\mathbb{C}[\lambda] \otimes$ $\left(P(\mathfrak{g}) / \mathcal{I}_{F}\right)$. Hence, for $A \in P(\mathfrak{g})$, if we denote the image of $A$ in $P(\mathfrak{g}) / \mathcal{I}_{F}$ by $[A]$ then

$$
\mathcal{W}(\mathfrak{g}, F)=\left(P(\mathfrak{g}) / \mathcal{I}_{F}\right)^{\operatorname{ad}_{\lambda} \mathfrak{n}}:=\left\{[A] \in P(\mathfrak{g}) / \mathcal{I}_{F} \mid\left\{n_{\lambda} A\right\} \subset \mathbb{C}[\lambda] \otimes \mathcal{I}_{F}\right\}
$$

is a well-defined vector superspace.

Remark 2.7. Since $P(\mathfrak{g}) / \mathcal{I}_{F} \simeq S(\mathbb{C}[\partial] \otimes \mathfrak{p})$ as superalgebras, each element in $P(\mathfrak{g}) / \mathcal{I}_{F}$ is uniquely represented by an element in $S(\mathbb{C}[\partial] \otimes \mathfrak{p})$. Hence, if there is no danger of confusion, we consider $\mathcal{W}(\mathfrak{g}, F)$ a subspace of $S(\mathbb{C}[\partial] \otimes \mathfrak{p})$.

Moreover, it is known that the vector superspace $\mathcal{W}(\mathfrak{g}, F)$ has a PVA structure.

Proposition 2.8. 8, 34, $\mathcal{W}(\mathfrak{g}, F)$ is a PVA endowed with the $\lambda$-bracket induced by the $\lambda$-bracket of the affine PVA $P(\mathfrak{g})$.

Definition 2.9. The PVA $\mathcal{W}(\mathfrak{g}, F)$ is called the classical $\mathrm{W}$-algebra associated with $\mathfrak{g}$ and $F$.

Now, let us define the conformal weight of $a \in \mathfrak{g}(i)$ by

$$
\Delta_{a}=1-i .
$$

Then $P(\mathfrak{g})$ has conformal weight decomposition via (2.9) and

$$
\Delta_{A B}=\Delta_{A}+\Delta_{B}, \quad \Delta_{\partial A}=A+1,
$$

where $A, B \in P(\mathfrak{g})$ are homogeneous elements with respect to the conformal weight.

Since the element $E \in \mathfrak{g}(1)$ in the $\mathfrak{s l}_{2}$-triple has a conformal weight of 0 , the ideal $\mathcal{I}_{F}$ of $P(\mathfrak{g})$ is homogeneous. Hence we can consider the conformal weight of the PVA $\mathcal{W}(\mathfrak{g}, F)$ induced from the conformal weight (2.9) of $P(\mathfrak{g})$.

We close this section by presenting crucial properties of a generating set of $\mathcal{W}(\mathfrak{g}, F)$.

Proposition 2.10. [8, 34] Let $\mathfrak{g}^{F}:=$ ker adF and $\mathcal{B}^{F}=\left\{q_{i} \mid i \in J^{F}\right\}$ be a homogeneous basis of $\mathfrak{g}^{F}$. Then there exists a set $\left\{w_{i} \mid i \in J^{F}\right\} \subset \mathcal{W}(\mathfrak{g}, F)$ such that

(i) $\mathcal{W}(\mathfrak{g}, F)=\mathbb{C}\left[\partial^{n} w_{i} \mid n \in \mathbb{Z}_{\geq 0}, i \in J^{F}\right]$ as differential algebras,

(ii) $\Delta_{w_{i}}=\Delta_{q_{i}}$,

(iii) $w_{i}=q_{i}+$ (total derivative part $)+($ degree $\geq 2$ part as the polynomial degree).

Remark 2.11. In Proposition 2.10, one can assume that $w_{i}-q_{i}$ does not have any monomial in $S\left(\mathbb{C}[\partial] \otimes \mathfrak{g}^{F}\right)$. If $w_{i}-q_{i}$ has a monomial $\partial^{n_{1}} q_{i_{1}} \partial^{n_{2}} q_{i_{2}} \cdots \partial^{n_{k}} q_{i_{k}} \in P\left(\mathfrak{g}^{F}\right)$ for $n_{1}, \cdots, n_{k} \in \mathbb{Z}_{\geq 0}$ and $i_{1}, \cdots, i_{k} \in J^{F}$ then $w_{i}$ can be replaced with $w_{i}-\partial^{n_{1}} w_{i_{1}} \partial^{n_{2}} w_{i_{2}} \cdots \partial^{n_{k}} w_{i_{k}}$. 


\section{Structures of Classical W-algebras associated with Lie superalgebras}

In this section, we find free generators of a classical affine $\mathrm{W}$-algebra $\mathcal{W}(\mathfrak{g}, F)$ and $\lambda$-brackets between them. We refer to [11] for the analogous results when $\mathfrak{g}$ is a Lie algebra.

As in the previous section, let $\mathfrak{g}$ be a simple Lie superalgebra with an $\mathfrak{s l}_{2}$-triple $(E, H, F)$ and even nondegenerate supersymmetric invariant bilinear form ( $\mid$ ) such that $\frac{1}{2}(H \mid H)=(E \mid F)=1$. Then $\mathfrak{g}=\bigoplus_{i \in \mathbb{Z} / 2} \mathfrak{g}(i)$ for the eigenspace $\mathfrak{g}(i)$ with respect to $\frac{H}{2}$.

According to the $\mathfrak{s l}_{2}$ representation theory, there are bases

$$
\left\{q_{j} \mid j \in J^{F}\right\}, \quad\left\{q^{j} \mid j \in J^{F}\right\}
$$

of $\mathfrak{g}^{F}:=\operatorname{ker} \operatorname{ad} F \subset \bigoplus_{i \leq 0} \mathfrak{g}(i)$ and $\mathfrak{g}^{E}:=\operatorname{ker} \operatorname{ad} E \subset \bigoplus_{i \geq 0} \mathfrak{g}(i)$ such that $\left(q^{i} \mid q_{j}\right)=\delta_{i, j}$. We assume the bases of $\mathfrak{g}^{F}$ and $\mathfrak{g}^{E}$ are homogeneous with respect to both parity and the $\frac{\mathbb{Z}}{2}$-grading. For simplicity of notation, we denote $s(i):=s\left(q_{i}\right)=s\left(q^{i}\right)$ for $i \in J^{F}$.

Again, by the representation of $\mathfrak{s l}_{2}$, we have

$$
\mathfrak{g}=\mathfrak{g}^{F} \oplus[E, \mathfrak{g}]
$$

and there is a homogeneous basis

$$
\mathcal{B}=\left\{q_{n}^{j}:=(\operatorname{ad} F)^{n} q^{j} \mid j \in J^{F}, n=0,1, \cdots, 2 \alpha_{j}\right\}
$$

of $\mathfrak{g}$, where $q^{j} \in \mathfrak{g}\left(\alpha_{j}\right)$.

Lemma 3.1. [11] Let $\mathcal{B}^{*}=\left\{q_{j}^{n} \mid j \in J^{F}, n=1, \cdots, 2 g_{j}\right\}$ be the dual basis of $\mathcal{B}$ such that $\left(q_{m}^{i} \mid q_{j}^{n}\right)=\delta_{i, j} \delta_{m, n}$. Then we have

$$
q_{j}^{n}=\frac{(-1)^{n}}{(n !)^{2}\left(\begin{array}{c}
2 \alpha_{j} \\
n
\end{array}\right)}(a d E)^{n} q_{j}
$$

Let us denote

$$
J_{k}^{F}:=\left\{(i, m) \in J^{F} \times \mathbb{Z}_{\geq 0} \mid q_{i}^{m} \in \mathfrak{g}(k) \text { or } q_{m}^{i} \in \mathfrak{g}(-k)\right\}
$$

and consider the following diffential algebra homomorphisms

$$
\begin{aligned}
& \pi: P(\mathfrak{p})=P\left(\mathfrak{g}^{F}\right) \otimes P\left(\left[E, \mathfrak{g}_{\leq-1 / 2}\right]\right) \rightarrow P\left(\mathfrak{g}^{F}\right), \quad A \otimes B \mapsto A, \\
& \rho: P(\mathfrak{g}) \rightarrow P(\mathfrak{p}), \quad a \mapsto \pi_{\leq \frac{1}{2}}(a)+(F \mid a) \text { for } a \in \mathfrak{g},
\end{aligned}
$$

where $P(\mathcal{E})=S(\mathbb{C}[\partial] \otimes \mathcal{E})$ for a vector superspace $\mathcal{E}$ and $\pi_{\leq 1 / 2}: \mathfrak{g} \rightarrow \mathfrak{g}_{\leq 1 / 2}$ is the projection map.

Lemma 3.2. 11

(1) If $(i, m) \in J_{t_{1}}^{F}$ and $(j, n) \in J_{t_{2}}^{F}$ for $t_{1}, t_{2} \in \frac{\mathbb{Z}}{2}$ then

$$
\rho\left\{q_{m \lambda}^{i} q_{j}^{n}\right\}= \begin{cases}0 & \text { if } t_{2}-t_{1}>1, \\ \delta_{i, j} \delta_{n, m+1} & \text { if } t_{2}-t_{1}=1, \\ {\left[q_{m}^{i}, q_{j}^{n}\right]+\delta_{i, j} \delta_{m, n} k \lambda} & \text { if } t_{2}-t_{1} \leq \frac{1}{2} .\end{cases}
$$

(2) If $\gamma \in P\left(\mathfrak{g}^{F}\right)\left(\mathbb{C}[\partial]\left[E, \mathfrak{g}_{\leq-1 / 2}\right]\right)$ satisfies

$$
\pi \rho\left\{a_{\lambda} \gamma\right\}=0 \quad \text { for any } \quad a \in \mathfrak{g}_{\geq 1 / 2}
$$

then $\gamma=0$. 
By Lemma 3.2, Proposition 2.10 and Remark 2.11, we have the unique differential algebra isomorphism

$$
\omega: P\left(\mathfrak{g}^{F}\right) \rightarrow \mathcal{W}(\mathfrak{g}, F), \quad a \mapsto \omega_{a} \text { for } a \in \mathfrak{g}^{F}
$$

such that $\omega_{a}=a+\gamma(a)+\gamma^{\geq 2}(a)$ where

$$
\gamma(a) \in P\left(\mathfrak{g}^{F}\right) \otimes\left(\mathbb{C}[\partial] \otimes\left[E, \mathfrak{g}_{\leq-1 / 2}\right]\right), \quad \gamma^{n}(a) \in P\left(\mathfrak{g}^{F}\right) \otimes\left(\mathbb{C}[\partial] \otimes\left[E, \mathfrak{g}_{\leq-1 / 2}\right]\right)^{\otimes n}
$$

and $\gamma^{\geq 2}(a)=\sum_{i \geq 2} \gamma^{i}(a)$.

Let us define the partial order $\prec$ on $\left(\bigcup_{k \in \frac{\mathbb{Z}}{2}} J_{k}^{F}\right) \cup \frac{\mathbb{Z}}{2}$ by

- $\left(j_{t}, n_{t}\right) \prec\left(j_{t+1}, n_{t+1}\right)$ if and only if $\left\{\begin{array}{l}\alpha_{t+1}-\alpha_{t} \geq 1 \text { where } \\ \left(j_{t}, n_{t}\right) \in J_{\alpha_{t}}^{F} \text { and }\left(j_{t+1}, n_{t+1}\right) \in J_{\alpha_{t+1}}^{F} \text {, }\end{array}\right.$

- $(j, n) \prec k$ (resp. $k \prec(j, n))$ if and only if $(j, n) \in J_{\beta}^{F}$ for $\beta \leq k-1$ (resp. $k \leq \beta-1$ )

for $k \in \frac{\mathbb{Z}}{2}$.

Theorem 3.3. Let $\pi_{\mathfrak{g}^{F}}: \mathfrak{g} \rightarrow \mathfrak{g}^{F}$ be the projection map and denote $a^{\sharp}:=\pi_{\mathfrak{g}^{F}}(a)$ for $a \in \mathfrak{g}$. For $q \in \mathfrak{g}^{F} \cap \mathfrak{g}(-\alpha)$, we have $\omega(q)=q+\gamma(q)+\gamma^{\geq 2}(q)$ where

$$
\begin{aligned}
\gamma(q)=\sum_{p \in \mathbb{Z}_{\geq 0}} \gamma_{p}(q)=\sum_{p \in \mathbb{Z}_{\geq 0}} \sum_{\substack{-\alpha-1 \prec\left(j_{0}, n_{0}\right) \prec \cdots \\
\ldots \prec\left(j_{p-1}, n_{p-1}\right) \prec\left(j_{p}, n_{p}\right) \prec \frac{1}{2}}} s\left(j_{0}\right)\left(\left[q, q_{n_{0}}^{j_{0}}\right]^{\sharp}-\left(q \mid q_{n_{0}}^{j_{0}}\right) k \partial\right) \\
\prod_{t=1, \cdots, p} s\left(j_{t}\right)\left(\left[q_{j_{t-1}}^{n_{t-1}+1}, q_{n_{t}}^{j_{t}}\right]^{\sharp}-\left(q_{j_{t-1}}^{n_{t-1}+1} \mid q_{n_{t}}^{j_{t}}\right) k \partial\right) q_{j_{p}}^{n_{p}+1} .
\end{aligned}
$$

Note that $\prod_{t=1, \cdots, p} A_{t}:=A_{1} A_{2} \cdots A_{p}$.

Proof. For $q_{m}^{i} \in \mathfrak{g}_{>0}$, we have

$$
\pi \rho\left(\left\{q_{m \lambda}^{i} q\right\}\right)=-s(q, i)\left(\left[q, q_{m}^{i}\right]^{\sharp}-\left(q \mid q_{m}^{i}\right) k \lambda\right)
$$


and

$$
\begin{aligned}
& \pi \rho\left(\left\{q_{m \lambda}^{i} \gamma_{p}(q)\right\}\right) \\
& =\sum_{\substack{-\alpha-1 \prec\left(j_{0}, n_{0}\right) \prec \cdots \\
\cdots \prec\left(j_{p}, n_{p}\right) \prec \frac{1}{2}}} s(q, i) s\left(q, j_{p}\right) s\left(j_{0}\right)\left(\left[q, q_{n_{0}}^{j_{0}}\right]^{\sharp}-\left(q \mid q_{n_{0}}^{j_{0}}\right) k(\partial+\lambda)\right) \\
& \prod_{t=1, \cdots, p} s\left(j_{t}\right)\left(\left[q_{j_{t-1}}^{n_{t-1}+1}, q_{n_{t}}^{j_{t}}\right]^{\sharp}-\left(q_{j_{t-1}}^{n_{t-1}+1} \mid q_{n_{t}}^{j_{t}}\right) k(\partial+\lambda)\right) \cdot \pi \rho\left\{q_{m \lambda}^{i} q_{j_{p}}^{n_{p}+1}\right\} \\
& =\sum_{\substack{\left(j_{0}, n_{0}\right) \prec\left(j_{1}, n_{1}\right) \prec \cdots \\
\cdots \prec\left(j_{p}, n_{p}\right)=(i, m)}} S_{1} \cdot\left(\left[q, q_{n_{0}}^{j_{0}}\right]^{\sharp}-\left(q \mid q_{n_{0}}^{j_{0}}\right) k(\partial+\lambda)\right) \\
& \prod_{t=1, \cdots, p-1}\left(\left[q_{j_{t-1}}^{n_{t-1}+1}, q_{n_{t}}^{j_{t}}\right]^{\sharp}-\left(q_{j_{t-1}}^{n_{t-1}+1} \mid q_{n_{t}}^{j_{t}}\right) k(\lambda+\partial)\right)\left(\left[q_{j_{p-1}}^{n_{p-1}+1}, q_{m}^{i}\right]^{\sharp}-\left(q_{j_{p-1}}^{n_{p-1}+1} \mid q_{m}^{i}\right) k \lambda\right) \\
& +\sum_{\substack{\left(j_{0}, n_{0}\right) \prec\left(j_{1}, n_{1}\right) \prec \cdots \\
\cdots \prec\left(j_{p}, n_{p}\right) \prec(i, m)}} S_{2} \cdot\left(\left[q, q_{n_{0}}^{j_{0}}\right]^{\sharp}-\left(q \mid q_{n_{0}}^{j_{0}}\right) k(\partial+\lambda)\right) \\
& \prod_{t=1, \cdots, p}\left(\left[q_{j_{t-1}}^{n_{t-1}+1}, q_{n_{t}}^{j_{t}}\right]^{\sharp}-\left(q_{j_{t-1}}^{n_{t-1}+1} \mid q_{n_{t}}^{j_{t}}\right) k(\lambda+\partial)\right)\left(\left[q_{j_{p-1}}^{n_{p-1}+1}, q_{m}^{i}\right]^{\sharp}-\left(q_{j_{p-1}}^{n_{p-1}+1} \mid q_{m}^{i}\right) k \lambda\right)
\end{aligned}
$$

where $S_{1}:=s(q, i) s\left(j_{0}\right) s\left(j_{1}\right) \cdots s\left(j_{p-1}\right)$ and $S_{2}:=-s(q, i) s\left(j_{0}\right) s\left(j_{1}\right) \cdots s\left(j_{p-1}\right) s\left(j_{p}\right)$. Hence $\pi \rho\left(\left\{q_{m \lambda}^{i} q+\right.\right.$ $\gamma(q)\})=0$. Since we know $\pi \rho\left(\left\{q_{m \lambda}^{i} \gamma^{\geq 2}(q)\right\}\right)=0$ and by Lemma 3.2 (2), we have proved the theorem.

Lemma 3.4. For any $t \in \frac{1}{2} \mathbb{Z}$, we have

$$
\sum_{(j, n) \in J_{-t}^{F}} s(j) q_{n}^{j} \otimes q_{j}^{n+1}=-\sum_{(i, m) \in J_{t-1}^{F}} q_{i}^{m+1} \otimes q_{m}^{i}
$$

Proof. Note that

- the both sides of (3.8) are in $[E, \mathfrak{g}(t-1)] \otimes[E, \mathfrak{g}(-t)]$,

- $[E, \mathfrak{g}(t)]$ is nondegenerately paired with $[F, \mathfrak{g}(-t)]$.

Hence it suffices to show that for $a \in \mathfrak{g}(-t+1)$ and $b \in \mathfrak{g}(t)$,

$$
\sum_{(j, n) \in J_{-t}^{F}} s(j)\left([F, a] \mid q_{n}^{j}\right)\left([F, b] \mid q_{j}^{n+1}\right)=-\sum_{(i, m) \in J_{t-1}^{F}}\left([F, a] \mid q_{i}^{m+1}\right)\left([F, b] \mid q_{m}^{i}\right) .
$$

One can check that

$$
\begin{aligned}
& \sum_{(j, n) \in J_{-t}^{F}} s(j)\left([F, a] \mid q_{n}^{j}\right)\left([F, b] \mid q_{j}^{n+1}\right)=\sum_{(j, n) \in J_{-t}^{F}}-\left(q_{n+1}^{j} \mid a\right)\left([F, b] \mid q_{j}^{n+1}\right)=-([F, b] \mid a), \\
& \sum_{(i, m) \in J_{t-1}^{F}}\left([F, a] \mid q_{i}^{m+1}\right)\left([F, b] \mid q_{m}^{i}\right)=-s(b)([F, a] \mid b)=([F, b] \mid a) .
\end{aligned}
$$


By the skew-symmetry of Lie superalgebras and the invariance of the bilinear form, we have proved the lemma.

Theorem 3.5. Let us take $a \in \mathfrak{g}\left(-t_{1}\right) \cap \mathfrak{g}^{F}$ and $b \in \mathfrak{g}\left(-t_{2}\right) \cap \mathfrak{g}^{F}$. Then

$$
\begin{aligned}
\left\{\omega(a)_{\lambda} \omega(b)\right\}=[a, b]+k \lambda(a \mid b) & \\
& -s(a, b) \sum_{p \in \mathbb{Z}_{\geq 0}} \sum_{\substack{-t_{2}-1 \prec\left(j_{0}, n_{0}\right) \prec \cdots \\
\cdots \prec\left(j_{p}, n_{p}\right) \prec t_{1}}} s\left(j_{0}\right) s\left(j_{1}\right) \cdots s\left(j_{p}\right)\left(\omega\left(\left[b, q_{n_{0}}^{j_{0}}\right]^{\sharp}\right)-\left(b \mid q_{n_{0}}^{j_{0}}\right) k(\lambda+\partial)\right) \\
& {\left[\prod_{t=1}^{p} \omega\left(\left[q_{j_{t-1}}^{n_{t-1}+1}, q_{n_{t}}^{j_{t}}\right]^{\sharp}\right)-\left(q_{j_{t-1}}^{n_{t-1}+1} \mid q_{n_{t}}^{j_{t}}\right) k(\lambda+\partial)\right]\left(\omega\left(\left[q_{j_{p}}^{n_{p}+1}, a\right]^{\sharp}\right)-\left(q_{j_{p}}^{n_{p}+1} \mid a\right) k \lambda\right) . }
\end{aligned}
$$

Proof. Let us denote

$$
\begin{aligned}
& \gamma_{p}(a)=\sum_{\substack{-t_{1}-1 \prec\left(i_{0}, m_{0}\right) \prec \\
\cdots \prec\left(i_{p}, m_{p}\right)}} s\left(i_{0}\right) s\left(i_{1}\right) \cdots s\left(i_{p}\right)\left(\left[a, q_{m_{0}}^{i_{0}}\right]^{\sharp}-\left(a \mid q_{m_{0}}^{i_{0}}\right) k \partial\right) \\
& \left(\left[q_{i_{0}}^{m_{0}+1}, q_{m_{1}}^{i_{1}}\right]^{\sharp}-\left(q_{i_{0}}^{m_{0}+1} \mid q_{m_{1}}^{i_{1}}\right) k \partial\right) \cdots\left(\left[q_{i_{p-1}}^{m_{p-1}+1}, q_{m_{p}}^{i_{p}}\right]^{\sharp}-\left(q_{i_{p-1}}^{m_{p-1}+1} \mid q_{m_{p}}^{i_{p}}\right) k \partial\right) q_{i_{p}}^{m_{p}+1}
\end{aligned}
$$

and

$$
\begin{aligned}
& \gamma_{s}(b)=\sum_{\substack{-t_{2}-1 \prec\left(j_{0}, n_{0}\right) \prec \\
\cdots \prec\left(j_{s}, n_{s}\right)}} s\left(j_{0}\right) s\left(j_{1}\right) \cdots s\left(j_{s}\right)\left(\left[b, q_{n_{0}}^{j_{0}}\right]^{\sharp}-\left(b \mid q_{n_{0}}^{j_{0}}\right) k \partial\right) \\
& \left(\left[q_{j_{0}}^{n_{0}+1}, q_{n_{1}}^{j_{1}}\right]^{\sharp}-\left(q_{j_{0}}^{n_{0}+1} \mid q_{n_{1}}^{j_{1}}\right) k \partial\right) \cdots\left(\left[q_{j_{s-1}}^{n_{s-1}+1}, q_{n_{s}}^{j_{s}}\right]^{\sharp}-\left(q_{j_{s-1}}^{n_{s-1}+1} \mid q_{n_{s}}^{j_{s}}\right) k \partial\right) q_{i_{s}}^{m_{s}+1} .
\end{aligned}
$$

To simplify the notation, let us denote

$$
\gamma_{p}(a)=\sum_{\left(i_{p}, m_{p}\right) \succ-t_{1}+p} A_{\left(i_{p}, m_{p}\right)}(\partial) q_{i_{p}}^{m_{p}+1} .
$$

Then

$$
\begin{aligned}
& \pi \rho\left\{\gamma_{p}(a)_{\lambda} \gamma_{s}(b)\right\} \\
& =\sum_{\substack{-t_{1}-1 \prec\left(i_{0}, m_{0}\right) \\
\cdots \prec\left(i_{p}, m_{p}\right)}} \sum_{\substack{-t_{2}-1 \prec\left(j_{0}, n_{0}\right) \\
\cdots \prec\left(j_{s}, n_{s}\right)}}\left(s\left(i_{0}\right) s\left(i_{1}\right) \cdots s\left(i_{p}\right)\right)\left(s\left(j_{0}\right) s\left(j_{1}\right) \cdots s\left(j_{s}\right)\right) \\
& \quad s(a, b) s\left(a, j_{s}\right) B_{\left(j_{s}, n_{s}\right)}(\lambda+\partial) \pi \rho\left(\left\{A_{\left(i_{p}, m_{p}\right)}(\partial) q_{i_{p}}^{m_{p}+1} q_{\lambda}^{n_{s}+1}\right\}\right),
\end{aligned}
$$

where

$$
\begin{aligned}
& B_{\left(j_{s}, n_{s}\right)}(\lambda+\partial)=\sum_{-t_{2}-1 \prec\left(j_{0}, n_{0}\right) \prec \cdots \prec\left(j_{s}, n_{s}\right)}\left(\left[b, q_{n_{0}}^{j_{0}}\right]^{\sharp}-\left(b \mid q_{n_{0}}^{j_{0}}\right) k(\lambda+\partial)\right) \\
&\left(\left[q_{j_{0}}^{n_{0}+1}, q_{n_{1}}^{j_{1}}\right]^{\sharp}-\left(q_{j_{0}}^{n_{0}+1} \mid q_{n_{1}}^{j_{1}}\right) k(\lambda+\partial)\right) \cdots\left(\left[q_{j_{s-1}}^{n_{s-1}+1}, q_{n_{s}}^{j_{s}}\right]^{\sharp}-\left(q_{j_{s-1}}^{n_{s-1}+1} \mid q_{n_{s}}^{j_{s}}\right) k(\lambda+\partial)\right) .
\end{aligned}
$$


Observe that

$$
\begin{gathered}
\sum_{\left(i_{p}, m_{p}\right) \succ-t_{1}+p} \pi \rho\left(\left\{A_{\left(i_{p}, m_{p}\right)}(\partial) q_{i_{p}}^{m_{p}+1} \lambda q_{j_{s}}^{n_{s}+1}\right\}\right) \\
=\sum_{-t_{1}-1 \prec\left(i_{0}, m_{0}\right) \prec \cdots \prec\left(i_{p}, m_{p}\right) \prec \frac{1}{2}} s\left(a q_{i_{0}}, q_{i_{0}} q_{j_{s}}\right) s\left(q_{i_{0}} q_{i_{1}}, q_{i_{1}} q_{j_{s}}\right) \cdots s\left(q_{i_{p-1}} q_{i_{p}}, q_{i_{p}} q_{j_{s}}\right) \\
\pi \rho\left(\left\{q_{i_{p}}^{m_{p}+1}{ }_{\lambda}^{n_{j_{s}}+1}\right\}\right)\left(\left[q_{i_{p-1}}^{m_{p-1}+1}, q_{m_{p}}^{i_{p}}\right]^{\sharp}+\left(q_{i_{p-1}}^{m_{p-1}+1} \mid q_{m_{p}}^{i_{p}}\right) k(\lambda+\partial)\right) \cdots \\
\cdots\left(\left[q_{i_{0}}^{m_{0}+1}, q_{m_{1}}^{i_{1}}\right]^{\sharp}+\left(q_{i_{0}}^{m_{0}+1} \mid q_{m_{1}}^{i_{1}}\right) k(\lambda+\partial)\right)\left(\left[a, q_{m_{0}}^{i_{0}}\right]^{\sharp}+\left(a \mid q_{m_{0}}^{i_{0}}\right) k \lambda\right) \\
=\sum_{-t_{1}-1 \prec\left(i_{0}, m_{0}\right) \prec \cdots \prec\left(i_{p}, m_{p}\right) \prec \frac{1}{2}}(-1)^{p^{2}} s\left(a, j_{s}\right) s\left(i_{0}\right) s\left(i_{1}\right) \cdots s\left(i_{p}\right) \\
\pi \rho\left(\left\{q_{j_{s}}^{n_{s}+1}-{ }_{-\lambda-\partial} q_{i_{p}}^{m_{p}+1}\right\}\right)\left(\left[q_{m_{p}}^{i_{p}}, q_{i_{p-1}}^{m_{p-1}+1}\right]^{\sharp}-\left(q_{m_{p}}^{i_{p}} \mid q_{i_{p-1}}^{m_{p-1}+1}\right) k(\lambda+\partial)\right) \cdots \\
\cdots\left(\left[q_{m_{1}}^{i_{1}}, q_{i_{0}}^{m_{0}+1}\right]^{\sharp}-\left(q_{m_{1}}^{i_{1}} \mid q_{i_{0}}^{m_{0}+1}\right) k(\lambda+\partial)\right)\left(\left[q_{m_{0}}^{i_{0}}, a\right]^{\sharp}-\left(q_{m_{0}}^{i_{0}} \mid a\right) k \lambda\right) .
\end{gathered}
$$

By Lemma 3.4.

$$
\begin{aligned}
& \text { (3.11) }=\sum_{-\frac{1}{2} \preceq\left(j_{s+1}, n_{s+1}\right) \prec \cdots \prec\left(j_{p+s+1}, n_{p+s+1}\right) \prec t_{1}}-s\left(a, j_{s}\right) \\
& \pi \rho\left(\left\{q_{j_{s}}^{n_{s}+1}-\lambda-\partial q_{n_{s+1}}^{j_{s+1}}\right\}\right)\left(\left[q_{j_{s+1}}^{n_{s+1}+1}, q_{n_{s+2}}^{j_{s+2}}\right]^{\sharp}-\left(q_{j_{s+1}}^{n_{s+1}+1} \mid q_{n_{s+2}}^{j_{s+2}}\right) k(\lambda+\partial)\right) \cdots \\
& \left(\left[q_{j_{s+p}}^{n_{s+p}+1}, q_{n_{s+p+1}}^{j_{s+p+1}}\right]^{\sharp}-\left(q_{j_{s+p}}^{n_{s+p}+1} \mid q_{n_{s+p+1}}^{j_{s+p+1}}\right) k(\lambda+\partial)\right)\left(\left[q_{j_{s+p+1}}^{n_{s+p+1}+1}, a\right]^{\sharp}-\left(q_{j_{s+p+1}}^{n_{s+p+1}+1} \mid a\right) k \lambda\right),
\end{aligned}
$$

where $\alpha \preceq(j, n)$ (resp, $\alpha \succeq(j, n))$ if and only if $q_{j}^{n} \in \mathfrak{g}_{\geq \alpha}$ (resp. $q_{j}^{n} \in \mathfrak{g}_{\leq \alpha}$ ) for $k \in \frac{\mathbb{Z}}{2}$.

Now, by (3.12), we have

$$
\pi \rho\left(\left\{q_{j_{s}}^{n_{s}+1}-\lambda-\partial q_{n_{s+1}}^{j_{s+1}}\right\}\right)= \begin{cases}{\left[q_{j_{s}}^{n_{s}+1}, q_{n_{s+1}}^{j_{s+1}}\right]^{\sharp}-\left(q_{j_{s}}^{n_{s}+1} \mid q_{n_{s+1}}^{j_{s+1}}\right) k(\lambda+\partial)} & \text { if }\left(j_{s}, n_{s}\right) \prec\left(j_{s+1}, n_{s+1}\right) \\
(-1)^{j_{s}+1} & \text { if }\left(\begin{array}{c}
\left(j_{s}, n_{s}\right)=\left(j_{s+1}, n_{s+1}\right), \\
\text { and } q_{j_{s}}^{n_{s}} \in \mathfrak{g}_{-1 / 2}
\end{array}\right) \\
0 & \text { otherwise. }\end{cases}
$$

Hence

$$
\pi \rho\left(\left\{\gamma_{p}(a)_{\lambda} \gamma_{s}(b)\right\}\right)=-s(a, b)\left(P_{p, s}+Q_{p, s}+R_{p, s}+S_{p, s}\right)
$$

for $p, s \in \mathbb{Z}_{\geq 0}$, where

$$
\begin{aligned}
P_{p, s}= & \sum_{\substack{\left(j_{s}, n_{s}\right) \in J_{-1 / 2}^{F} \\
-t_{2} \preceq\left(j_{0}, n_{0}\right) \prec \cdots \prec\left(j_{s+p+1}, n_{s+p+1}\right) \prec t_{1}}} s\left(j_{0}\right) s\left(j_{1}\right) \cdots s\left(j_{s+p+1}\right) \\
\left(\left[b, q_{n_{0}}^{j_{0}}\right]^{\sharp}-\left(b \mid q_{n_{0}}^{j_{0}}\right) k(\lambda+\partial)\right)\left(\left[q_{j_{0}}^{n_{0}+1}, q_{n_{1}}^{j_{1}}\right]^{\sharp}-\left(q_{j_{0}}^{n_{0}+1} \mid q_{n_{1}}^{j_{1}}\right) k(\lambda+\partial)\right) & \left.\left(\left[q_{j_{1}}^{n_{1}+1}, q_{n_{2}}^{j_{2}}\right]^{\sharp}-\left(q_{j_{1}}^{n_{1}+1} \mid q_{n_{2}}^{j_{2}}\right) k(\lambda+\partial)\right) \cdots\left(q_{j_{s+p+1}}^{n_{s+p+1}+1}, a\right]^{\sharp}-\left(q_{j_{s+p+1}}^{n_{s+p+1}+1} \mid a\right) k(\lambda)\right),
\end{aligned}
$$




$$
\begin{aligned}
& Q_{p, s}= \sum_{\substack{\left(j_{s+1}, n_{s+1}\right) \in J_{-1 / 2}^{F},-t_{2} \preceq\left(j_{0}, n_{0}\right) \prec \cdots \prec\left(j_{s+p+1}, n_{s+p+1}\right) \prec t_{1} \\
\left(\left[b, q_{n_{0}}^{j_{0}}\right]^{\sharp}-\left(b \mid q_{n_{0}}^{j_{0}}\right) k(\lambda+\partial)\right)\left(\left[q_{j_{0}}^{n_{0}+1}, q_{n_{1}}^{j_{1}}\right]^{\sharp}-\left(q_{j_{0}}^{n_{0}+1} \mid q_{n_{1}}^{j_{1}}\right) k(\lambda+\partial)\right)}} \quad s\left(j_{1}\right) \cdots s\left(j_{s+p+1}\right) \\
&\left(\left[q_{j_{1}}^{n_{1}+1}, q_{n_{2}}^{j_{2}}\right]^{\sharp}-\left(q_{j_{1}}^{n_{1}+1} \mid q_{n_{2}}^{j_{2}}\right) k(\lambda+\partial)\right) \cdots\left(\left[q_{j_{s+p+1}}^{n_{s+p+1}+1}, a\right]^{\sharp}-\left(q_{j_{s+p+1}}^{n_{s+p+1}+1} \mid a\right) k(\lambda)\right), \\
& R_{p, s}=\sum_{\left(j_{s}, n_{s}\right),\left(j_{s+1}, n_{s+1}\right) \notin J_{-1 / 2}^{F},} s\left(j_{0}\right) s\left(j_{1}\right) \cdots s\left(j_{s+p+1}\right) \\
&-t_{2} \preceq\left(j_{0}, n_{0}\right) \prec \cdots \prec\left(j_{s+p+1}, n_{s+p+1} \prec t_{1}\right. \\
&\left(\left[b, q_{n_{0}}^{j_{0}}\right]^{\sharp}-\left(b \mid q_{n_{0}}^{j_{0}}\right) k(\lambda+\partial)\right)\left(\left[q_{j_{0}}^{n_{0}+1}, q_{n_{1}}^{j_{1}}\right]^{\sharp}-\left(q_{j_{0}}^{n_{0}+1} \mid q_{n_{1}}^{j_{1}}\right) k(\lambda+\partial)\right) \\
&\left(\left[q_{j_{1}}^{n_{1}+1}, q_{n_{2}}^{j_{2}}\right]^{\sharp}-\left(q_{j_{1}}^{n_{1}+1} \mid q_{n_{2}}^{j_{2}}\right) k(\lambda+\partial)\right) \cdots\left(\left[q_{j_{s+p+1}}^{n_{s+p+1}+1}, a\right]^{\sharp}-\left(q_{j_{s+p+1}}^{n_{s+p+1}+1} \mid a\right) k(\lambda)\right),
\end{aligned}
$$

and

$$
\begin{gathered}
\sum_{p, s}=\sum_{\begin{array}{c}
\left(j_{s}, n_{s}\right),\left(j_{s+1}, n_{s+1}\right) \in J_{-1 / 2}^{F}, \\
-t_{2} \preceq\left(j_{0}, n_{0}\right) \prec \cdots \prec\left(j_{s}, n_{s}\right) \\
\prec\left(j_{s+2}, n_{s+2}\right) \prec \cdots \prec\left(j_{s+p+1}, n_{s+p+1}\right) \prec t_{1} \\
\left(\left[b, q_{n_{0}}^{j_{0}}\right]^{\sharp}-\left(b \mid q_{n_{0}}^{j_{0}}\right) k(\lambda+\partial)\right)\left(\left[q_{j_{0}}^{n_{0}+1}, q_{n_{1}}^{j_{1}}\right]^{\sharp}-\left(q_{j_{0}}^{n_{0}+1} \mid q_{n_{1}}^{j_{1}}\right) k(\lambda+\partial)\right) \cdots
\end{array}}-\left(s\left(j_{0}\right) s\left(j_{1}\right) \cdots s\left(j_{s-1}\right)\right)\left(s\left(j_{s+1}\right) s\left(j_{s+2}\right) \cdots s\left(j_{s+p+1}\right)\right) \\
\left(\left[q_{j_{s-1}}^{n_{s-1}+1}, q_{n_{s}}^{j_{s}}\right]^{\sharp}-\left(q_{j_{s-1}}^{n_{s-1}+1} \mid q_{n_{s}}^{j_{s}}\right) k(\lambda+\partial)\right)\left(\left[q_{j_{s+1}}^{n_{s+1}+1}, q_{n_{s+2}}^{j_{s+2}}\right]^{\sharp}-\left(q_{j_{s+1}}^{n_{s+1}+1} \mid q_{n_{s+2}}^{j_{s+2}}\right) k(\lambda+\partial)\right) \\
\cdots\left(\left[q_{j_{s+p+1}}^{n_{s+p+1}+1}, a\right]^{\sharp}-\left(q_{j_{s+p+1}}^{n_{s+p+1}+1} \mid a\right) k(\lambda)\right) .
\end{gathered}
$$

In addition, we have

$$
\begin{aligned}
& \pi \rho\left(\left\{a_{\lambda} \gamma_{s}(b)\right\}\right)=-s(a, b)\left(P_{-1, s}+R_{-1, s}\right) \\
& \pi \rho\left(\left\{\gamma_{p}(a)_{\lambda} b\right\}\right)=-s(a, b)\left(Q_{p,-1}+R_{p,-1}\right)
\end{aligned}
$$

where $P_{-1, s}, R_{-1, s}, Q_{p,-1}, R_{p,-1}$ are obtained by letting $p=-1$ or $s=-1$ in (3.13), (3.14) or (3.15). Observe

$$
P_{p, s}=-S_{p+1, s}=Q_{p+1, s-1}
$$

and thus

$$
\begin{aligned}
& \sum_{N=0}^{\infty} \sum_{p+s=N} \pi \rho\left\{a+\gamma_{p}(a)_{\lambda} b+\gamma_{s}(b)\right\}=[a, b]+k \lambda(a \mid b) \\
&-s(a, b) \sum_{\substack{-k \preceq\left(j_{0}, n_{0}\right) \prec \cdots \\
\cdots \prec\left(j_{s+p+1}, n_{s+p+1}\right) \prec h}} s\left(j_{0}\right) s\left(j_{1}\right) \cdots s\left(j_{s+p+1}\right) \\
&\left(\left[b, q_{n_{0}}^{j_{0}}\right]^{\sharp}-\left(b \mid q_{n_{0}}^{j_{0}}\right) k(\lambda+\partial)\right)\left(\left[q_{j_{0}}^{n_{0}+1}, q_{n_{1}}^{j_{1}}\right]^{\sharp}-\left(q_{j_{0}}^{n_{0}+1} \mid q_{n_{1}}^{j_{1}}\right) k(\lambda+\partial)\right) \cdots \\
&\left(\left[q_{j_{s+p}}^{n_{s+p}+1}, q_{n_{s+p+1}}^{j_{s+p+1}}\right]^{\sharp}-\left(q_{j_{s+p}}^{n_{s+p}+1} \mid q_{n_{s+p+1}}^{j_{s+p+1}}\right) k(\lambda+\partial)\right)\left(\left[q_{j_{s+p+1}}^{n_{s+p+1}+1}, a\right]^{\sharp}-\left(q_{j_{s+p+1}}^{n_{s+p+1}+1} \mid a\right) k(\lambda)\right),
\end{aligned}
$$

which implies the theorem. 


\section{Supersymmetric Poisson vertex algebras}

In this section, we recall the notion of supersymmetric vertex algebras. For more details, we refer to [17.

Definition 4.1. Let $\mathcal{R}$ be a Lie superalgebra with an odd operator $D: \mathcal{R} \rightarrow \mathcal{R}$, that is $D\left(\mathcal{R}_{\bar{i}}\right) \subset \mathcal{R}_{\bar{i}+\overline{1}}$ for $i=0,1$.

(1) A $\chi$-bracket on a $\mathbb{C}[D]$-module $\mathcal{R}$ is a linear map

$$
[\chi]: \mathcal{R} \otimes \mathcal{R} \rightarrow \mathbb{C}[\chi] \otimes \mathcal{R}
$$

where $\chi$ is an odd indeterminate and $p(a)+p(b)+1=p\left(\left[a_{\chi} b\right]\right)$ for any homogeneous elements $a, b \in \mathcal{R}$. In this paper, we usually denote

$$
\left[a_{\chi} b\right]=\sum_{n \in \mathbb{Z}_{\geq 0}} \chi^{n} a_{[n]} b
$$

for $a_{[n]} b \in \mathcal{R}$.

(2) A $\mathbb{C}[D]$-module $\mathcal{R}$ is called a supersymmetric (SUSY) Lie conformal algebra (LCA) if it satisfies the following properties:

- $\left(\right.$ sesquilinearity) $\left[D a_{\chi} b\right]=\chi\left[a_{\chi} b\right],\left[a_{\chi} D b\right]=-s(a)(D+\chi)\left[a_{\chi} b\right]$,

- (skew-symmetry) $\left[a_{\chi} b\right]=s(a, b)\left[b_{-\chi-D} a\right]$ where

$$
\left[b_{-\chi-D} a\right]=\sum_{n \in \mathbb{Z}_{\geq 0}}(-D-\chi)^{n} b_{[n]} a
$$

and $\mathbb{C}[\chi] \otimes \mathcal{R}$ is a $\mathbb{C}[D]$-module via

$$
\chi D+D \chi=-2 \chi^{2} .
$$

- (Jacobi identity) $\left[a_{\chi}\left[b_{\gamma} c\right]\right]=-s(a)\left[\left[a_{\chi} b\right]_{\chi+\gamma} c\right]-s(a, b) s(a) s(b)\left[b_{\gamma}\left[a_{\chi} c\right]\right]$, where $\gamma$ is an odd indeterminate supercommuting with $\chi$. The LHS of the Jacobi identity is computed via

$$
\left[a_{\chi} \gamma^{n} b_{[n]} c\right]=(-s(a) \gamma)^{n}\left[a_{\chi} b_{[n]} c\right]
$$

and the RHS is computed via

$$
\begin{aligned}
& {\left[\chi^{n} a_{[n]} b_{\chi+\gamma} c\right]=(-\chi)^{n}\left[a_{[n]} b_{\chi+\gamma} c\right],} \\
& {\left[b_{\gamma} \chi^{n} a_{[n]} c\right]=(-s(b) \chi)^{n}\left[b_{\gamma} a_{[n]} c\right] .}
\end{aligned}
$$

Definition 4.2. A tuple $(\mathcal{P},\{\chi\}, 1, \cdot, D)$ is called a $S U S Y P V A$ if it satisfies:

- $(\mathcal{P}, 1, \cdot, D)$ is a unital differential supercommutative algebra with odd derivation $D$,

- $(\mathcal{P},\{\chi\}, D)$ is a SUSY PVA,

- (Leibniz rule) $\left\{a_{\chi} b c\right\}=\left\{a_{\chi} b\right\} c+s(b, c)\left\{a_{\chi} c\right\} b$ for any $a, b, c \in \mathcal{P}$.

The (right) Leibniz rule and skew-symmetry of SUSY LCAs induce the left Leibniz rule

$$
\left\{a b_{\chi} c\right\}=s(b, c)\left\{a_{\chi+D} c\right\}_{\rightarrow} b+s(a, b c)\left\{b_{\chi+D} c\right\} \rightarrow a,
$$

where

$$
\left\{a_{\chi+D} c\right\}_{\rightarrow} b=\sum_{n \in \mathbb{Z}_{\geq 0}} s(a c)^{n} a_{[n]} c(\chi+D)^{n} b .
$$

Proposition 4.3. A SUSY PVA is a PVA. More precisely, if $(\mathcal{P},\{\chi\}, 1, \cdot, D)$ is a SUSY PVA then $\left(\mathcal{P},\left\{{ }_{\lambda}\right\}, 1, \cdot, \partial=D^{2}\right)$ is a PVA via

$$
a_{(n)} b:=(-1)^{n} a_{[2 n+1]} b,
$$

where $\left\{a_{\lambda} b\right\}=\sum_{n \in \mathbb{Z}_{\geq 0}} \lambda^{n} a_{(n)} b$ and $\left\{a_{\chi} b\right\}=\sum_{n \in \mathbb{Z}_{\geq 0}} \chi^{n} a_{[n]} b$ for $a, b \in \mathcal{P}$. 
Proof. By the sesquilineaity of SUSY LCA $\mathcal{P}$,

$$
D^{2} a_{[2 n+1]} b=a_{[2 n-1]} b \quad \text { and } \quad a_{[2 n+1]} D^{2} b=D^{2}\left(a_{[2 n+1]} b\right)-a_{[2 n-1]} b .
$$

for $a, b \in \mathcal{P}$ and $n \in \mathbb{Z}_{\geq 1}$. Thus, (4.3) implies that

$$
\partial a_{(n)} b=-a_{(n-1)} b \quad \text { and } \quad a_{(n)} \partial b=\partial\left(a_{(n)} b\right)+a_{(n-1)} b,
$$

which are equivalent to the sesquilinearity of LCA.

By the skew-symmetry of SUSY LCA $\mathcal{P}$,

$$
a_{[2 n+1]} b=\sum_{m \geq n} s(a, b)(-1)^{m+1}\left(\begin{array}{c}
m \\
n
\end{array}\right) D^{2 m-2 n}\left(b_{[2 m+1]} a\right)
$$

for $a, b \in \mathcal{P}$ and $n \in \mathbb{Z}_{>0}$. More precisely, the LHS and RHS are the coefficients of $\chi^{2 n+1}$ in $\left\{a_{\chi} b\right\}$ and $s(a, b)\left\{b_{-\chi-D} a\right\}$, respectively. Due to (4.3) and $D^{2}=\partial$, (4.4) is equivalent to

$$
a_{(n)} b=-s(a, b) \sum_{m \geq n}\left(\begin{array}{c}
m \\
n
\end{array}\right)(-1)^{m} \partial^{m-n}\left(b_{(m)} a\right) .
$$

One can check that the LHS and RHS of (4.5) are coefficients of $\lambda^{n}$ in $\left\{a_{\lambda} b\right\}$ and $-s(a, b)\left\{b_{-\lambda-\partial} a\right\}$.

To see the Jacobi identity of $\mathcal{P}$ as a LCA, one has to show that

$$
a_{(n)}\left(b_{(m)} c\right)=\sum_{j \geq m}\left(\begin{array}{c}
j \\
m
\end{array}\right)\left(a_{(n+m-j)} b\right)_{(j)} c+s(a, b) b_{(m)}\left(a_{(n)} c\right)
$$

for $a, b, c \in \mathcal{P}$ and $n, m \in \mathbb{Z}_{\geq 0}$. In fact, (4.6) is the coefficient of $\chi^{2 n+1} \gamma^{2 m+1}$ in

$$
\left[a_{\chi}\left[b_{\gamma} c\right]\right]=-s(a)\left[\left[a_{\chi} b\right]_{\chi+\gamma} c\right]-s(a, b) s(a) s(b)\left[b_{\gamma}\left[a_{\chi} c\right]\right] .
$$

Hence the Jacobi identity of LCA $\mathcal{P}$ holds.

Finally, a similar argument works for the right Leibniz rule of $\mathcal{P}$. Hence the SUSY PVA structure of $\mathcal{P}$ naturally induces the PVA structure of $\mathcal{P}$.

\section{Proposition 4.4. 4}

(1) Let $V$ be a vector superspace and $\mathcal{R}(V):=\mathbb{C}[D] \otimes V$ be the $\mathbb{C}[D]$-module freely generated by $V$. Let

$$
[\chi]: V \otimes V \rightarrow \mathbb{C}[\chi] \otimes(\mathcal{R}(V))
$$

be an odd linear map satisfying the skew-symmetry and Jacobi identity of the $\chi$-brackets. Then the bracket (4.7) can be extended to the bracket on $\mathcal{R}(V)$ via the sesquilinearity and $\mathcal{R}(V)$ endowed with the $\chi$-bracket is a SUSY PVA.

(2) Let $\left(\mathcal{R},\left[{ }_{\chi}\right]\right)$ be a SUSY LCA. Then the supersymmetric algebra $\mathcal{P}:=S(\mathcal{R})$ endowed with the $\chi$-bracket $\left\{\chi_{\chi}\right\}$ extended from the $\chi$-bracket on $\mathcal{R}$ via the Leibniz rule is a SUSY PVA.

Let us fix the vector superspace $V$ with a basis $\mathcal{B}=\left\{u_{i} \mid i \in I\right\}$, which is homogenous with respect to the parity and let

$$
\mathcal{R}:=\mathbb{C}[D] \otimes V, \quad \mathcal{P}:=S(\mathcal{R}) .
$$

Then $\mathcal{R}$ is the vector superspace with the basis $\left\{u_{i}^{[m]}:=D^{m}\left(u_{i}\right) \mid i \in I, m \in \mathbb{Z}_{\geq 0}\right\}$ and $\mathcal{P}$ is the superalgebra of polynomials in $\left\{u_{i}^{[m]} \mid i \in I, m \in \mathbb{Z}_{\geq 0}\right\}$. For simplicity of notation, we write $s(i):=s\left(u_{i}\right), s(i, j):=s\left(u_{i}, u_{j}\right)$ for $i, j \in I$.

Definition 4.5. Let $\frac{\partial}{\partial u_{i}^{[m]}}$ be the derivation on $\mathcal{P}$ of parity $p\left(u_{i}\right)+\bar{m} \in \mathbb{Z} / 2 \mathbb{Z}$ such that

$$
\frac{\partial}{\partial u_{i}^{[m]}}\left(u_{j}^{[n]}\right)=\delta_{i, j} \delta_{m, n}
$$


One can check the commutator $\left[\frac{\partial}{\partial u_{i}^{[m]}}, D\right]$ is (i) a derivation of parity $p\left(u_{i}\right)+m+1$ and (ii) $\left[\frac{\partial}{\partial u_{i}^{[m]}}, D\right]\left(u_{j}^{[n]}\right)=\delta_{i, j} \delta_{m-1, n}$. Since such derivation is unique, we have

$$
\left[\frac{\partial}{\partial u_{i}^{[m]}}, D\right]=\frac{\partial}{\partial u_{i}^{[m-1]}}
$$

where $\frac{\partial}{\partial u_{i}^{[-1]}}:=0$.

Theorem 4.6 (Master formula). 4] Let $\mathcal{P}$ be a SUSY PVA. Then for $a, b \in \mathcal{P}$,

$$
\begin{aligned}
&\left\{a_{\chi} b\right\}=\sum_{i, j \in I, m, n \in \mathbb{Z}_{\geq 0}} S\left(a_{(i, m)}, b_{(j, n)}\right) b_{(j, n)}\left\{u_{i}^{[m]}{ }_{\chi}+D u_{j}^{[n]}\right\}_{\rightarrow} a_{(i, m)} \\
&=\sum_{i, j \in I, m, n \in \mathbb{Z}_{\geq 0}} S\left(a_{(i, m)}, b_{(j, n)}\right)(-1)^{n+m n+\frac{(m+1) m}{2}} s(i)^{n+m} s(j)^{m} \\
& b_{(j, n)}(\chi+D)^{n}\left\{u_{i \chi+D} u_{j}\right\}_{\rightarrow}(\chi+D)^{m} a_{(i, m)}
\end{aligned}
$$

where

- $a_{(i, m)}:=\frac{\partial}{\partial u_{i}^{[m]}}$ a and $b_{(j, n)}:=\frac{\partial}{\partial u_{j}^{[n]}} b$

- $S\left(a_{(i, m)}, b_{(j, n)}\right):=s\left(b_{(j, n)}\right) s\left(b_{(j, n)}, u_{j}^{[n]} a\right) s\left(a_{(i, m)}, u_{j}^{[n]}\right)$.

Example 4.7 (Affine SUSY PVA). Let $\mathfrak{g}$ be a Lie superalgebra with an even invariant supersymmetric bilinear form $(\mid)$ and let $\overline{\mathfrak{g}}=\{\bar{a} \mid a \in \mathfrak{g}\}$ be the vector superspace such that $p(a)=1+p(\bar{a})$ for a homogeneous element $a \in \mathfrak{g}$. Consider the $\chi$-bracket

$$
[\chi]:(\overline{\mathfrak{g}} \oplus \mathbb{C} K) \otimes(\overline{\mathfrak{g}} \oplus \mathbb{C} K) \rightarrow \mathbb{C}[\chi] \otimes(\overline{\mathfrak{g}} \oplus \mathbb{C} K)
$$

such that

$$
\left[\bar{a}_{\chi} \bar{b}\right]=s(a)(\overline{[a, b]}+\chi K(a \mid b)), \quad\left[K_{\chi} \bar{a}\right]=\left[K_{\chi} K\right]=0
$$

for $a, b, \in \mathfrak{g}$.

Let $\mathcal{R}:=\mathbb{C}[D] \otimes \overline{\mathfrak{g}} \oplus \mathbb{C} K$ be a $\mathbb{C}[D]$-module via $D\left(D^{n} \bar{a}\right)=D^{n+1} \bar{a}$ and $D(K)=0$. The bracket (4.9) can be extended to the bracket on $\mathcal{R}$ using the sesquilinearity. Thus, $\mathcal{R}$ is a SUSY LCA called the affine SUSY LCA associated with $\mathfrak{g}$.

By Proposition 4.4, the supercommutative differential algebra $\mathcal{P}_{K}(\overline{\mathfrak{g}})=S(\mathcal{R})$ is a SUSY PVA endowed with the bracket $\{\chi\}$ induced from the bracket on $\mathcal{R}$ and the Leibniz rule. In addition, the supersymmetric differential algebra $\mathcal{P}(\overline{\mathfrak{g}}):=S(\mathbb{C}[D] \otimes \overline{\mathfrak{g}}) \simeq P_{K} /(K-k) P_{K}$ is also a SUSY PVA called the affine SUSY PVA associated with $\overline{\mathfrak{g}}$ and $k \in \mathbb{C}$.

\section{SUSY CLASSICAL W-ALGEBRAS}

5.1. First definition of SUSY classical W-algebras. Let $\mathfrak{g}$ be a simple finite Lie superalgebra with a subalgebra $\mathfrak{s}=\operatorname{Span}_{\mathbb{C}}\{E, e, H, f, F\}$ isomorphic to osp $(1 \mid 2)$ such that

- $(E, H, F)$ is an $\mathfrak{s l}_{2}$-triple,

- $e, f$ are odd elements,

- $[H, e]=e,[H, f]=-f,[e, e]=2 E,[f, f]=-2 F,[e, f]=-H,[F, e]=f,[E, f]=e$.

We also assume that $\mathfrak{g}$ is endowed with an even nondegenerate invariant supersymmetric bilinear form ( | ) such that

$$
(H \mid H)=2(E \mid F)=2, \quad(e \mid f)=-(f \mid e)=-2 .
$$


Since $H$ is in $\mathfrak{s l}_{2}$, the Lie superalgebra $\mathfrak{g}$ can be decomposed into $\mathfrak{g}=\bigoplus_{i \in \mathbb{Z} / 2} \mathfrak{g}(i)$ where $\mathfrak{g}(i)=$ $\{a \in \mathfrak{g} \mid[H, a]=2 i a\}$.

Recall the affine SUSY PVA $\mathcal{P}(\overline{\mathfrak{g}})$ in Example 4.7 and consider the super Lie subalgebra

$$
\mathfrak{n}=\mathfrak{g}_{>0} \text {. }
$$

Let $\mathcal{I}_{f}$ be the differential algebra ideal of $\mathcal{P}(\overline{\mathfrak{g}})$ generated by $\{\bar{n}-(f \mid n) \mid n \in \mathfrak{n}\}$. Then the quotient space $\mathcal{P}(\overline{\mathfrak{g}}) / \mathcal{I}_{f}$ is a well-defined differential algebra and let $\operatorname{ad}_{\chi} \overline{\mathfrak{n}}: \mathcal{P}(\overline{\mathfrak{g}}) / \mathcal{I}_{f} \rightarrow \mathbb{C}[\chi] \otimes \mathcal{P}(\overline{\mathfrak{g}}) / \mathcal{I}_{f}$ be defined by

$$
\operatorname{ad}_{\chi} \bar{n}([A])=\sum_{m \in \mathbb{Z}_{\geq 0}} \chi^{m}\left[\bar{n}_{[m]} A\right] \in \mathbb{C}[\chi] \otimes\left(\mathcal{P}(\overline{\mathfrak{g}}) / \mathcal{I}_{f}\right)
$$

where $[A]$ and $\left[\bar{n}_{[m]} A\right]$ are the images of $A$ and $\bar{n}_{[m]} A$ in $\mathcal{P}(\overline{\mathfrak{g}}) / \mathcal{I}_{f}$, respectively. Since $\left\{\bar{n}_{\chi} \mathcal{I}_{f}\right\} \in$ $\mathbb{C}[\chi] \otimes \mathcal{I}_{f}$, the vector superspace

$$
\mathcal{W}(\overline{\mathfrak{g}}, f):=\left(\mathcal{P}(\overline{\mathfrak{g}}) / \mathcal{I}_{f}\right)^{\operatorname{ad}_{\chi} \overline{\mathfrak{n}}}=\left\{\bar{A} \in \mathcal{P}(\overline{\mathfrak{g}}) / \mathcal{I}_{f} \mid \operatorname{ad}_{\chi}(\bar{A})=0\right\}
$$

is well-defined.

Proposition 5.1. The vector superspace $\mathcal{W}(\overline{\mathfrak{g}}, f)$ is a SUSY PVA endowed with the bracket induced from the $\chi$-bracket of the affine SUSY PVA $\mathcal{P}(\overline{\mathfrak{g}})$.

Proof. Let us take $A, B \in \mathcal{P}(\overline{\mathfrak{g}})$ such that $[A],[B] \in \mathcal{W}(\overline{\mathfrak{g}}, f)$ and $n \in \mathfrak{n}$. To show that $\mathcal{W}(\overline{\mathfrak{g}}, f)$ is a differential superalgebra, it is sufficient to show that $\left\{\bar{n}_{\chi} A B\right\}$ and $\left\{\bar{n}_{\chi} D A\right\}$ are in $\mathbb{C}[\chi] \otimes \mathcal{I}_{f}$. Since

$$
\begin{aligned}
& \left\{\bar{n}_{\chi} A B\right\}=\left\{\bar{n}_{\chi} A\right\} B+s(A, B)\left\{\bar{n}_{\chi} B\right\} A \\
& \left\{\bar{n}_{\chi} D A\right\}=s(n)(D+\chi)\left\{\bar{n}_{\chi} A\right\}
\end{aligned}
$$

and $\left\{\bar{n}_{\chi} A\right\},\left\{\bar{n}_{\chi} B\right\} \in \mathbb{C}[\chi] \otimes \mathcal{I}_{f}$, both equations in (5.3) are in $\mathbb{C}[\chi] \otimes \mathcal{I}_{f}$. In order to show that $\mathcal{W}(\overline{\mathfrak{g}}, f)$ is SUSY LCA, we need to show that $\left\{\bar{n}_{\chi}\left\{A_{\gamma} B\right\}\right\} \in \mathbb{C}[\chi, \gamma] \otimes \mathcal{I}_{f}$. Since $\left\{I_{\chi} A\right\}$ and $\left\{I_{\chi} B\right\}$ are in $\mathbb{C}[\chi] \otimes \mathcal{I}_{f}$ for any $I \in \mathcal{I}_{f}$, we have

$$
\left\{\bar{n}_{\chi}\left\{A_{\gamma} B\right\}\right\}=s(n)\left\{\left\{\bar{n}_{\chi} A\right\}_{\chi+\gamma} B\right\}+s(A, n) s(n)\left\{A_{\gamma}\left\{\bar{n}_{\chi} B\right\}\right\} \in \mathbb{C}[\chi, \gamma] \otimes \mathcal{I}_{f} .
$$

Hence $\mathcal{W}(\overline{\mathfrak{g}}, f)$ is a SUSY PVA.

Definition 5.2. The SUSY PVA $\mathcal{W}(\overline{\mathfrak{g}}, f)$ is called the SUSY classical W-algebra associated with $\overline{\mathfrak{g}}$ and $f$.

5.2. Second definition of SUSY classical W-algebras. In this section, we introduce SUSY classical W-algebras via SUSY classical BRST complexes. We refer to [27, 28] for the construction of SUSY W-algebras using SUSY quantum BRST complexes. The SUSY classical BRST complexes introduced in this section can be understood as classical limits of the quantum complexes.

Consider the following two SUSY PVAs:

(I) Let $j_{\overline{\mathfrak{g}}}=\left\{j_{\bar{a}} \mid a \in \mathfrak{g}\right\}$ be isomorphic to $\overline{\mathfrak{g}}$ as vector superspaces. Consider the supersymmetric algebra

$$
S\left(\mathbb{C}[D] \otimes j_{\overline{\mathfrak{g}}}\right)
$$

generated by $\mathbb{C}[D] \otimes j_{\overline{\mathfrak{g}}}$. Define the SUSY PVA structure on $S\left(\mathbb{C}[D] \otimes j_{\overline{\mathfrak{g}}}\right)$ by

$$
\left[j_{\bar{a}} j_{\bar{b}}\right]=s(a, \bar{b}) j_{\overline{[a, b]}}+k \chi(a \mid b) \text { for } a, b \in \mathfrak{g}, k \in \mathbb{C} .
$$

According to Proposition $4.4, S\left(\mathbb{C}[D] \otimes j_{\overline{\mathfrak{g}}}\right)$ is a SUSY PVA. 
(II) For the subspace $\mathfrak{n}=\bigoplus_{i>0} \mathfrak{g}(i)$, let $\phi_{\mathfrak{n}} \simeq \mathfrak{n} \subset \mathfrak{g}$ and $\phi^{\overline{\mathfrak{n}}_{-}} \simeq \overline{\mathfrak{n}}_{-} \subset \overline{\mathfrak{g}}$ as vector superspaces. The supersymmetric algebra

$$
S\left(\mathbb{C}[D] \otimes\left(\phi_{\mathfrak{n}} \oplus \phi^{\overline{\mathfrak{n}}}\right)\right)
$$

endowed with the $\chi$-bracket defined by

$$
\left[\phi^{\bar{a}}{ }_{\chi} \phi_{b}\right]=\left[\phi_{b} \chi \phi^{\bar{a}}\right]=(a \mid b)
$$

is a SUSY PVA.

Remark 5.3. The SUSY PVA (I) is isomorphic to the affine SUSY PVA $\mathcal{P}(\overline{\mathfrak{g}})$ by the map

$$
j_{\bar{a}} \mapsto \begin{cases}\mathrm{i} \bar{a} & \text { if } a \in \mathfrak{g} \text { is odd, } \\ \bar{a} & \text { if } a \in \mathfrak{g} \text { is even, }\end{cases}
$$

for the imaginary number $i \in \mathbb{C}$.

Consider the SUSY PVA

$$
\mathcal{C}(\overline{\mathfrak{g}}, f)=S\left(\mathbb{C}[D] \otimes j_{\overline{\mathfrak{g}}}\right) \otimes S\left(\mathbb{C}[D] \otimes\left(\phi_{\mathfrak{n}} \oplus \phi^{\overline{\mathfrak{n}}}\right)\right)
$$

which is the tensor product of two SUSY PVAs (I) and (II). Let us denote the index set by $I_{>}$ such that

$$
\left\{u_{\alpha}\right\}_{\alpha \in I_{>}} \text {and }\left\{u^{\alpha}\right\}_{\alpha \in I_{>}}
$$

are bases of $\mathfrak{n}$ and $\mathfrak{n}_{-}=\mathfrak{g}_{<0}$, respectively, satisfying $\left(u^{\alpha} \mid u_{\beta}\right)=\delta_{\alpha, \beta}$. For simplicity of notation, let

$$
s(\alpha, \beta)=s\left(u_{\alpha}, u_{\beta}\right), \quad s(\beta)=s\left(u_{\beta}\right)
$$

for $\alpha, \beta \in I_{>}$and let

$$
j_{\bar{\alpha}}=j_{\bar{u}_{\alpha}}, \quad \phi_{\alpha}=\phi_{u_{\alpha}}, \quad \phi^{\bar{\alpha}}=\phi^{\bar{u}^{\alpha}}, \quad \phi_{a}=\phi_{\pi_{+}(a)}, \quad \phi^{\bar{a}}=\phi^{\overline{\pi_{-}(a)}}
$$

for the projection maps $\pi_{+}: \mathfrak{g} \rightarrow \mathfrak{n}, \pi_{-}=\mathfrak{g} \rightarrow \mathfrak{n}_{-}$.

Take the element

$$
d^{c}=\sum_{\alpha \in I_{>}}\left(j_{\bar{\alpha}}-c\left(f \mid u_{\alpha}\right)\right) \phi^{\bar{\alpha}}+\frac{1}{2} \sum_{\alpha, \beta \in I_{>}} s(\alpha, \beta) s(\beta) \phi_{\left[u_{\alpha}, u_{\beta}\right]} \phi^{\bar{\beta}} \phi^{\bar{\alpha}} \in \mathcal{C}(\overline{\mathfrak{g}}, f),
$$

for $c \in \mathbb{C}$. If there is no need to emphasize the value $c \in \mathbb{C}$, we simply denote $d^{c}$ as $d$.

\section{Proposition 5.4.}

(1) The $\chi$-brackets between $d$ and the elements in $\mathcal{C}(\overline{\mathfrak{g}}, f)$ are

$$
\begin{aligned}
& \left\{d_{\chi} j_{\bar{a}}\right\}=\sum_{\alpha \in I_{>}} s(\alpha, a) s(\alpha) \phi^{\bar{\alpha}} j_{\left[u_{\alpha}, a\right]}-\sum_{\alpha \in I_{>}} s(\alpha) k(\chi+D) \phi^{\bar{\alpha}}\left(u_{\alpha} \mid a\right), \\
& \left\{d_{\chi} \phi^{\bar{\alpha}}\right\}=\frac{1}{2} \sum_{\beta \in I_{>}} s(\alpha, \beta) s(\beta) \phi^{\bar{\beta}} \phi^{\overline{\left[u_{\beta}, u^{\alpha}\right]}}, \\
& \left\{d_{\chi} \phi_{\alpha}\right\}=-s(\alpha) j_{\bar{\alpha}}-c\left(f \mid u_{\alpha}\right)+\sum_{\beta \in I_{>}} s(\alpha, \beta) s(\beta) \phi^{\bar{\beta}} \phi_{\left[u_{\beta}, u_{\alpha}\right]} .
\end{aligned}
$$

(2) Recall that $d_{[0]} A=\left.\left\{d_{\chi} A\right\}\right|_{\chi=0}$ for $A \in \mathcal{C}(\overline{\mathfrak{g}}, f)$. We have $d_{[0]}^{2}=0$. 
Proof. (1) We have

$$
\begin{aligned}
\left\{d_{\chi} j_{\bar{a}}\right\} & =\sum_{\alpha \in I_{>}}\left\{\left(j_{\bar{\alpha}}-c\left(f \mid u_{\alpha}\right)\right) \phi_{\chi}^{\bar{\alpha}} j_{\bar{a}}\right\}=\sum_{\alpha \in I_{>}}\left\{j_{\bar{a}}-\chi-D\left(j_{\bar{\alpha}}-c\left(f \mid u_{\alpha}\right)\right) \phi^{\bar{\alpha}}\right\} \\
& =\sum_{\alpha \in I_{>}} s(a, \alpha) s(a)\left(j_{\overline{\left[a, u_{\alpha}\right]}}+k\left(a \mid u_{\alpha}\right)(-\chi-D)\right) \phi^{\bar{\alpha}} \\
& =\sum_{\alpha \in I_{>}}\left(s(\alpha, a) s(\alpha) \phi^{\bar{\alpha}} j_{\overline{\left[u_{\alpha}, a\right]}}-s(\alpha) k(\chi+D) \phi^{\bar{\alpha}}\left(u_{\alpha} \mid a\right)\right) .
\end{aligned}
$$

Here we used the skew-symmetry and Leibniz rule of the $\chi$-bracket and the fact that $d$ is an even element. We can also check that

$$
\begin{aligned}
\left\{d_{\chi} \phi^{\bar{\alpha}}\right\} & =\frac{1}{2} \sum_{\beta, \gamma \in I_{>}}\left\{\phi_{-\chi-D}^{\bar{\alpha}} s(\beta, \gamma) s(\gamma) \phi_{\left[u_{\beta}, u_{\gamma}\right]} \phi^{\bar{\gamma}} \phi^{\bar{\beta}}\right\} \\
& =\frac{1}{2} \sum_{\beta, \gamma \in I_{>}} s(\beta, \gamma) s(\gamma)\left(u^{\alpha} \mid\left[u_{\beta}, u_{\gamma}\right]\right) \phi^{\bar{\gamma}} \phi^{\bar{\beta}}=\frac{1}{2} \sum_{\alpha, \beta \in I_{>}} s(\alpha, \beta) s(\beta) \phi^{\bar{\beta}} \phi^{\overline{\left[u_{\beta}, u^{\alpha}\right]}}
\end{aligned}
$$

and

$$
\begin{aligned}
\left\{d_{\chi} \phi_{\alpha}\right\} & =\left\{\phi_{\alpha-\chi-D} d\right\} \\
& =-s(\alpha) \bar{u}_{\alpha}-c\left(f \mid u_{\alpha}\right)+\frac{1}{2} \sum_{\beta, \gamma \in I_{>}}\left\{\phi_{\alpha-\chi-D} s(\beta, \gamma) s(\gamma) \phi_{\left[u_{\beta}, u_{\gamma}\right]} \phi^{\bar{\gamma}} \phi^{\bar{\beta}}\right\} \\
& =-s(\alpha) \bar{u}_{\alpha}-c\left(f \mid u_{\alpha}\right)+\sum_{\beta \in I_{>}} s(\alpha, \beta) s(\beta) \phi^{\bar{\beta}} \phi_{\left[u_{\beta}, u_{\alpha}\right]} .
\end{aligned}
$$

(2) By the Jacobi identity,

$$
d_{[0]}\left(d_{[0]} A\right)=-\left(\left(d_{[0]} d\right)_{[0]} A\right)-d_{[0]}\left(d_{[0]} A\right)
$$

for any $A \in \mathcal{C}(\overline{\mathfrak{g}}, f, c)$. Hence it is sufficient to show $d_{[0]} d=0$. By (1), one can verify that

- $\sum_{\alpha, \beta \in I_{>}}\left\{j_{\bar{\alpha}} \phi^{\bar{\alpha}}{ }_{\chi} j_{\bar{\beta}} \phi^{\bar{\beta}}\right\}=\sum_{\alpha, \beta \in I_{>}} s(\alpha, \beta) s(\alpha) j_{\overline{\left[u_{\alpha}, u_{\beta}\right]}} \phi^{\bar{\beta}} \phi^{\bar{\alpha}}$,

- $\sum_{\alpha, \beta, \gamma \in I_{>}}\left\{j_{\bar{\alpha}} \phi^{\bar{\alpha}}{ }_{\chi} s(\beta, \gamma) s(\gamma) \phi_{\left[u_{\beta}, u_{\gamma}\right]} \phi^{\bar{\gamma}} \phi^{\bar{\beta}}\right\}=\sum_{\beta, \gamma \in I_{>}}-s(\beta, \gamma) s(\beta) j_{\left[u_{\beta}, u_{\gamma}\right]} \phi^{\bar{\gamma}} \phi^{\bar{\beta}}$.

Hence, for $d_{2}:=\sum_{\alpha, \beta \in I_{>}} s(\alpha, \beta) s(\beta) \phi_{\left[u_{\alpha}, u_{\beta}\right]} \phi^{\bar{\beta}} \phi^{\bar{\alpha}}$, we have

$$
\left\{d_{\chi} d\right\}=\frac{1}{4}\left\{d_{2 \chi} d_{2}\right\} .
$$

By the master formula,

$$
\left\{d_{2 \chi} d_{2}\right\}=\sum_{\gamma, \delta \in I_{>}}\left(\left\{d_{2 \chi} s(\gamma, \delta) s(\delta) \phi_{\left[u_{\gamma}, u_{\delta}\right]}\right\} \phi^{\bar{\delta}} \phi^{\bar{\gamma}}+2\left\{d_{2 \chi} s(\gamma) s(\delta) \phi^{\bar{\delta}}\right\} \phi_{\left[u_{\gamma}, u_{\delta}\right]} \phi^{\bar{\gamma}}\right) .
$$

Since

- $\sum_{\gamma, \delta \in I_{>}}\left\{d_{2 \chi} s(\gamma, \delta) s(\delta) \phi_{\left[u_{\gamma}, u_{\delta}\right]}\right\} \phi^{\bar{\delta}} \phi^{\bar{\gamma}}=\sum_{\alpha, \gamma, \delta \in I_{>}} s(\alpha) s(\delta) \phi_{\left[u_{\alpha},\left[u_{\gamma}, u_{\delta}\right]\right]} \phi^{\bar{\alpha}} \phi^{\bar{\gamma}} \phi^{\bar{\delta}}$

- $\sum_{\gamma, \delta \in I_{>}}\left\{d_{2 \chi} s(\gamma) s(\delta) \phi^{\bar{\delta}}\right\} \phi_{\left[u_{\gamma}, u_{\delta}\right]} \phi^{\bar{\gamma}}=-\frac{1}{2} \sum_{\alpha, \gamma, \delta \in I_{>}} s(\alpha) s(\delta) \phi_{\left[u_{\alpha},\left[u_{\gamma}, u_{\delta}\right]\right]} \phi^{\bar{\alpha}} \phi^{\bar{\gamma}} \phi^{\bar{\delta}}$,

we have $\left\{d_{2 \chi} d_{2}\right\}=0$. Thus $\left\{d_{\chi} d\right\}=0$ and $d_{[0]}^{2}=0$.

By Proposition $5.4(2)$, the map $d_{[0]}$ is an odd differential on $\mathcal{C}(\overline{\mathfrak{g}}, f)$.

\section{Proposition 5.5.}

(1) If $A \in \mathcal{C}(\overline{\mathfrak{g}}, f)$ is in the image of $d_{[0]}$ then $D A$ is also in the image of $d_{[0]}$.

(2) If $B \in \operatorname{ker} d_{[0]}$ then $d_{[0]}(A) B$ for $A \in \mathcal{C}(\overline{\mathfrak{g}}, f)$ is in the image of $d_{[0]}$.

(3) If $B \in \operatorname{ker} d_{[0]}$ and $A \in \mathcal{C}(\overline{\mathfrak{g}}, f, c)$ then both $\left\{d_{[0]}(A)_{\chi} B\right\}$ and $\left\{B_{\chi} d_{[0]}(A)\right\}$ are in $\mathbb{C}[\chi] \otimes$ $i m\left(d_{[0]}\right)$. 
Proof. (1) By the sesquilinearity of SUSY PVAs, $d_{[0]}(D A)=-D\left(d_{[0]} A\right)$.

(2) By the Leibniz rule of SUSY PVAs,

$$
d_{[0]}(A B)=s(A) A d_{[0]}(B)+s(A, B) s(B) B d_{[0]}(A)=d_{[0]}(A) B .
$$

(3) By the Jacobi identity of SUSY PVAs,

$$
\begin{aligned}
& d_{[0]}\left(\left\{A_{\chi} B\right\}\right)=-\left\{d_{[0]}(A)_{\chi} B\right\}-s(A)\left\{A_{\chi} d_{[0]}(B)\right\}=-\left\{d_{[0]}(A)_{\chi} B\right\}, \\
& d_{[0]}\left(\left\{B_{\chi} A\right\}\right)=-\left\{d_{[0]}(B)_{\chi} A\right\}-s(B)\left\{B_{\chi} d_{[0]}(A)\right\}=-s(B)\left\{B_{\chi} d_{[0]}(A)\right\} .
\end{aligned}
$$

Hence we have proved the proposition.

Theorem 5.6. The SUSY PVA structure of $\mathcal{C}(\overline{\mathfrak{g}}, f)$ induces the SUSY PVA structure on

$$
\mathcal{W}_{B R}^{c}(\overline{\mathfrak{g}}, f):=H\left(\mathcal{C}(\overline{\mathfrak{g}}, f), d_{[0]}^{c}\right) .
$$

Proof. By Proposition 5.5 (1), (2) and (3), $\mathcal{W}_{\mathrm{BR}}^{c}(\overline{\mathfrak{g}}, f)$ is closed under derivation $D$, the supercommutative product and the $\chi$-bracket.

We also simply denote the algebra $(5.12)$ by $\mathcal{W}_{\mathrm{BR}}(\overline{\mathfrak{g}}, f)$ if the constant $c$ does not play a crucial role.

5.3. Equivalence of two definitions of SUSY classical W-algebras. In this section, we compare SUSY PVAs defined in Theorem 5.6] and Definition [5.2, We refer to [5] for the detailed properties of bigraded complexes adopted in this section.

Recall the complex $\mathcal{C}(\overline{\mathfrak{g}}, f)$ in (5.4) and the differential $d_{[0]}$ for $d$ in (5.7). In order to see the structure of $\mathcal{W}_{\mathrm{BR}}(\overline{\mathfrak{g}}, f)$, we consider the building block

$$
J_{\bar{a}}=j_{\bar{a}}-\sum_{\beta \in I_{>}} s(a, \beta) s(a) s(\beta) \phi^{\bar{\beta}} \phi_{\left[u_{\beta}, a\right]} \in \mathcal{C}(\overline{\mathfrak{g}}, f)
$$

for $a \in \mathfrak{g}$. Then

$$
\begin{array}{r}
\left\{d_{\chi} J_{\bar{a}}\right\}=\sum_{\gamma \in I_{>}}\left(s(a, \gamma) s(\gamma) \phi^{\bar{\gamma}} j_{\overline{\left[u_{\gamma}, a\right]}}-k s(\gamma) D \phi^{\bar{\gamma}}\left(u_{\gamma} \mid a\right)\right) \\
-\sum_{\beta \in I_{>}} s(a, \beta) s(a) s(\beta)\left\{d_{\chi} \phi^{\bar{\beta}} \phi_{\left[u_{\beta}, a\right]}\right\} .
\end{array}
$$

In the last term of the previous equation,

$$
\begin{aligned}
\sum_{\beta \in I_{>}}\left\{d_{\chi} \phi^{\bar{\beta}} \phi_{\left[u_{\beta}, a\right]}\right\}= & \frac{1}{2} \sum_{\beta, \gamma \in I_{>}} s(\gamma, \beta) s(\gamma) \phi^{\bar{\gamma}} \phi^{\overline{\left[u_{\gamma}, u^{\beta}\right]}} \phi_{\left[u_{\beta}, a\right]} \\
& +\sum_{\beta \in I_{>}} \phi^{\bar{\beta}}\left((-1)^{a} \overline{\pi_{+}\left[u_{\beta}, a\right]}-(-1)^{a} c\left(f \mid \pi_{+}\left[u_{\beta}, a\right]\right)\right) \\
& -\sum_{\beta, \gamma \in I_{>}} s\left(\gamma,\left[u_{\beta}, a\right]\right) s(\gamma) s(\beta) \phi^{\bar{\beta}} \phi^{\bar{\gamma}} \phi_{\left[u_{\gamma}, \pi_{+}\left[u_{\beta}, a\right]\right]} .
\end{aligned}
$$


Since

$$
\begin{aligned}
& \sum_{\beta, \gamma \in I_{>}} s(\gamma, \beta) s(\gamma) \phi^{\bar{\gamma}} \phi^{\overline{\left[u_{\gamma}, u^{\beta}\right]}} \phi_{\left[u_{\beta}, a\right]}=\sum_{\beta, \gamma, \delta \in I_{>}} s(\gamma, \delta) \phi^{\bar{\gamma}} \phi^{\bar{\delta}}\left(\left[u_{\gamma}, u^{\beta}\right] \mid u_{\delta}\right) \phi_{\left[u_{\beta}, a\right]} \\
= & \sum_{\gamma, \delta \in I_{>}}\left(s(\gamma, \delta) s(\delta) \phi^{\bar{\delta}} \phi^{\bar{\gamma}} \phi_{\left[u_{\gamma},\left[u_{\delta}, a\right]\right]}+s(\gamma, \delta) s(\gamma) \phi^{\bar{\gamma}} \phi^{\bar{\delta}} \phi_{\left[u_{\delta},\left[u_{\gamma}, a\right]\right]}\right) \\
= & \sum_{\gamma, \delta \in I_{>}} 2 s(\gamma, \delta) s(\delta) \phi^{\bar{\delta}} \phi^{\bar{\gamma}} \phi_{\left[u_{\gamma},\left[u_{\delta}, a\right]\right]}
\end{aligned}
$$

we have

$$
\begin{aligned}
& \left\{d_{\chi} J_{\bar{a}}\right\} \\
= & \sum_{\beta \in I_{>}}\left(s(a, \beta) s(\beta) \phi^{\bar{\beta}} j_{\overline{\pi_{\leq 0}\left[u_{\beta}, a\right]}}-s(\beta) k(D+\chi) \phi^{\bar{\beta}}\left(u_{\beta} \mid a\right)+s(a, \beta) s(\beta) \phi^{\bar{\beta}} c\left(f \mid\left[u_{\beta}, a\right]\right)\right) \\
& -\sum_{\beta, \gamma \in I_{>}} s(a, \beta) s(a, \gamma) s(\beta, \gamma) s(a) s(\gamma) \phi^{\bar{\beta}} \phi^{\bar{\gamma}} \phi_{\overline{\left[u_{\gamma}, \pi_{\leq 0}\left[u_{\beta}, a\right]\right]}} \\
= & \sum_{\beta \in I_{>}} s(a, \beta) s(\beta) \phi^{\bar{\beta}}\left(J_{\overline{\pi_{\leq 0}\left[u_{\beta}, a\right]}}+c\left(f \mid\left[u_{\beta}, a\right]\right)\right)-\sum_{\beta \in I_{>}} s(\beta) k D \phi^{\bar{\beta}}\left(u_{\beta} \mid a\right),
\end{aligned}
$$

where $\pi_{\leq 0}: \mathfrak{g} \rightarrow \mathfrak{g}_{\leq 0}$ is the projection map.

The $\chi$-bracket between two building blocks is

$$
\begin{aligned}
& \left\{J_{\bar{a}} \chi J_{\bar{b}}\right\}=\left\{j_{\bar{a}_{\chi}} j_{\bar{b}}\right\}+\sum_{\beta, \gamma \in I_{>}}\left\{s(a, \beta) s(a) s(\beta) \phi^{\bar{\beta}} \phi_{\left[u_{\beta}, a\right] \chi} s(b, \gamma) s(b) s(\gamma) \phi^{\bar{\gamma}} \phi_{\left[u_{\gamma}, b\right]}\right\} \\
& =\left\{j_{\bar{a}_{\chi}} j_{\bar{b}}\right\}-\sum_{\beta, \gamma \in I_{>}} s(a, \beta) s(a, b) s(b, \beta) s(b) s(\beta)\left(\phi^{\bar{\beta}} \phi_{\left[\pi_{+}\left[u_{\beta}, a\right], b\right]}-s(a, b) \phi^{\bar{\beta}} \phi_{\left[\pi_{+}\left[u_{\beta}, b\right], a\right]}\right) \\
& =s(a, b) s(a) j_{\overline{[a, b]}}+k(D+\chi)(a \mid b)-\sum_{\beta \in I_{>}} s(a, b) s(a, \beta) s(b, \beta) s(b) s(\beta) \phi^{\bar{\beta}} \phi_{\left[u_{\beta},[a, b]\right]} \\
& +\sum_{\beta, \gamma \in I_{>}} s(a, \beta) s(a, b) s(b, \beta) s(b) s(\beta)\left(\phi^{\bar{\beta}} \phi_{\left[\pi_{\leq 0}\left[u_{\beta}, a\right], b\right]}-s(a, b) \phi^{\bar{\beta}} \phi_{\left[\pi_{\leq 0}\left[u_{\beta}, b\right], a\right]}\right) .
\end{aligned}
$$

Hence

$$
\left\{J_{\bar{a} \chi} J_{\bar{b}}\right\}=s(a, b) s(a) J_{\overline{[a, b]}}+k(\chi+D)(a \mid b)
$$

if $a, b \in \mathfrak{g}_{\leq 0}$ or $a, b \in \mathfrak{g}_{>0}$. Let

$$
r_{+}=\phi_{\mathfrak{n}} \oplus d_{[0]}\left(\phi_{\mathfrak{n}}\right), \quad r_{-}=J_{\overline{\mathfrak{g}}_{\leq 0}} \oplus \phi^{\overline{\mathfrak{n}}_{-}}, \quad \mathcal{R}_{+}=\mathbb{C}[D] \otimes r_{+}, \quad \mathcal{R}_{-}=\mathbb{C}[D] \otimes r_{-} .
$$

Lemma 5.7 (Künneth theorem). (e.g., see [5]) Let $U_{1}, U_{2}$ and $U=U_{1} \otimes U_{2}$ be superalgebras and $d_{1}$ and $d_{2}$ be differentials on $U_{1}$ and $U_{2}$, respectively. If $d=d_{1} \otimes 1+1 \otimes d_{2}$ then

$$
H(U, d)=H\left(U_{1}, d_{1}\right) \otimes H\left(U_{2}, d_{2}\right) .
$$

Further, if $d$ is a linear differential map on a vector superspace $V$ then

$$
H(S(V), d) \simeq S(H(V, d))
$$

where $S(V)$ is the supersymmetric algebra generated by $V$ and $d$ on $S(V)$ is the differential map induced from that on $V$.

Proposition 5.8. Recall that $\mathcal{W}_{B R}(\overline{\mathfrak{g}}, f)=H\left(\mathcal{C}(\overline{\mathfrak{g}}, f), d_{[0]}\right)$. 
(1) $\mathcal{C}(\overline{\mathfrak{g}}, f)=S\left(\mathcal{R}_{+}\right) \otimes S\left(\mathcal{R}_{-}\right)$.

(2) $\left.d_{[0]}\right|_{S\left(R_{+}\right)} \subset S\left(R_{+}\right)$and $\left.d_{[0]}\right|_{S\left(\mathcal{R}_{-}\right)} \subset S\left(\mathcal{R}_{-}\right)$.

(3) $\mathcal{W}_{B R}(\overline{\mathfrak{g}}, f) \simeq H\left(S\left(\mathcal{R}_{-}\right), d_{[0]}=\left.d_{[0]}\right|_{S\left(\mathcal{R}_{-}\right)}\right)$.

Proof. (1) It is enough to check that every element in $\mathfrak{g}, \phi_{\mathfrak{n}}, \phi^{\overline{\mathfrak{n}}_{-}}$are in $S\left(\mathcal{R}_{+}\right) \otimes S\left(\mathcal{R}_{-}\right)$.

(2) Since $d_{[0]}\left(\phi_{n}\right) \in S\left(\mathcal{R}_{+}\right)$and $d_{[0]}^{2}\left(\phi_{n}\right)=0$, we see that $\left.d_{[0]}\right|_{S\left(\mathcal{R}_{+}\right)} \subset S\left(\mathcal{R}_{+}\right)$. By (5.17) and (5.8), we obtain $\left.d_{[0]}\right|_{S\left(\mathcal{R}_{-}\right)} \subset S\left(\mathcal{R}_{-}\right)$.

(3) Using the Künneth theorem, we have

$$
\begin{aligned}
\mathcal{W}_{\mathrm{BR}}(\mathfrak{g}, f, k) & \simeq H\left(S\left(\mathcal{R}_{+}\right),\left.d_{[0]}\right|_{S\left(\mathcal{R}_{+}\right)}\right) \otimes H\left(S\left(\mathcal{R}_{-}\right),\left.d_{[0]}\right|_{S\left(\mathcal{R}_{-}\right)}\right) \\
& \simeq H\left(S\left(\mathcal{R}_{-}\right),\left.d_{[0]}\right|_{S\left(\mathcal{R}_{-}\right)} .\right.
\end{aligned}
$$

Here, we used $H\left(S\left(\mathcal{R}_{+}\right),\left.d_{[0]}\right|_{S\left(\mathcal{R}_{+}\right)}\right)=S\left(H\left(\mathcal{R}_{+},\left.d_{[0]}\right|_{\mathcal{R}_{+}}\right)\right)=\mathbb{C}$.

Now we observe the cohomology $H\left(S\left(\mathcal{R}_{-}\right), d_{[0]}\right)$ by defining $\frac{1}{2} \mathbb{Z}$-bigrading on $S\left(\mathcal{R}_{-}\right)$:

$$
\operatorname{gr}\left(J_{a}\right)=\left(g_{a},-g_{a}\right), \quad \operatorname{gr}\left(\phi^{\beta}\right)=\left(-g_{\beta}+1 / 2, g_{\beta}+1 / 2\right)
$$

where $a \in \mathfrak{g}\left(g_{a}\right)$ and $u_{\beta} \in \mathfrak{g}\left(g_{\beta}\right)$ and $\operatorname{gr}(D)=(0,0)$ which induces the bigrading on $S\left(\mathcal{R}_{-}\right)$. We write

$$
S\left(\mathcal{R}_{-}\right)^{n}:=\left\{A \in S\left(\mathcal{R}_{-}\right) \mid \operatorname{gr}(A)=(p, q) \text { and } p+q=n\right\}
$$

for $n \in \mathbb{Z}_{\geq 0}$. Consider the decreasing filtration $\left\{F^{p} S\left(\mathcal{R}_{-}\right) \mid p \in \mathbb{Z} / 2\right\}$ such that

$$
F^{p} S\left(\mathcal{R}_{-}\right):=\operatorname{Span}_{\mathbb{C}}\left\{A \in S\left(\mathcal{R}_{-}\right) \mid \operatorname{gr}(A)=\left(p^{\prime}, q\right) \text { for } p^{\prime} \geq p\right\} \text {. }
$$

Then $F^{p} S\left(\mathcal{R}_{-}\right)=\bigoplus_{n \in \mathbb{Z}} F^{p} S\left(\mathcal{R}_{-}\right)^{n}$ where $F^{p} S\left(\mathcal{R}_{-}\right)^{n}:=F^{p} S\left(\mathcal{R}_{-}\right) \cap S\left(\mathcal{R}_{-}\right)^{n}$. One can check that

$$
\left.d_{[0]}\right|_{S\left(\mathcal{R}_{-}\right)}\left(F^{p} S\left(\mathcal{R}_{-}\right)^{n}\right) \subset F^{p} S\left(\mathcal{R}_{-}\right)^{n+1} .
$$

Thus the cohomology $H\left(S\left(\mathcal{R}_{-}\right), d_{[0]}\right)$ is also $\mathbb{Z}_{\geq 0}$-graded and $\mathbb{Z} / 2$-filtered space with

$$
F^{p} H^{n}\left(S\left(\mathcal{R}_{-}\right), d_{[0]}\right)=\frac{\operatorname{ker}\left(\left.d_{[0]}\right|_{F^{p}} S\left(\mathcal{R}_{-}\right)^{n}\right)}{\operatorname{im}\left(\left.d_{[0]}\right|_{F^{p}} S\left(\mathcal{R}_{-}\right)^{n-1}\right)}
$$

and let

$$
\operatorname{gr}^{p, q} H\left(S\left(\mathcal{R}_{-}\right), d_{[0]}\right):=\frac{F^{p} H^{p+q}\left(S\left(\mathcal{R}_{-}\right), d_{[0]}\right)}{F^{p+\frac{1}{2}} H^{p+q}\left(S\left(\mathcal{R}_{-}\right), d_{[0]}\right)} .
$$

Denote

$$
\operatorname{gr} S\left(\mathcal{R}_{-}\right):=\bigoplus_{p, q \in \mathbb{Z} / 2} \operatorname{gr}^{p, q} S\left(\mathcal{R}_{-}\right)
$$

where $\operatorname{gr}^{p, q} S\left(\mathcal{R}_{-}\right)=F^{p} S\left(\mathcal{R}_{-}\right)^{p+q} / F^{p+\frac{1}{2}} S\left(\mathcal{R}_{-}\right)^{p+q}$ and consider the differential $d^{\mathrm{gr}}$ on $\operatorname{gr} S\left(\mathcal{R}_{-}\right)$ induced from $d_{[0]}$. Then

$$
d^{\mathrm{gr}}\left(J_{a}\right)=\sum_{\beta \in S} s(a, \beta) s(\beta) \phi^{\beta}\left(f \mid\left[u_{\beta}, a\right]\right), \quad d^{\mathrm{gr}}\left(\phi^{\beta}\right)=0
$$

and the cohomology $H\left(\operatorname{gr} S\left(\mathcal{R}_{-}\right), d^{\text {gr }}\right)$ is $\frac{\mathbb{Z}}{2}$-bigraded via

$$
H^{p, q}\left(\operatorname{gr} S\left(\mathcal{R}_{-}\right), d^{\mathrm{gr}}\right)=\frac{\operatorname{ker}\left(d^{\mathrm{gr}}: \operatorname{gr}^{p, q} S\left(\mathcal{R}_{-}\right) \rightarrow \operatorname{gr}^{p, q+1} S\left(\mathcal{R}_{-}\right)\right)}{\operatorname{im}\left(d^{\mathrm{gr}}: \operatorname{gr}^{p, q-1} S\left(\mathcal{R}_{-}\right) \rightarrow \operatorname{gr}^{p, q} S\left(\mathcal{R}_{-}\right)\right)}
$$


Lemma 5.9. The complex $\left(S\left(\mathcal{R}_{-}\right), d_{[0]}\right)$ is good, i.e., $H^{p, q}\left(\operatorname{gr} S\left(\mathcal{R}_{-}, d^{g r}\right), d^{g r}\right)=0$ for any $p, q \in$ $\mathbb{Z} / 2$ such that $p+q \neq 0$. Moreover,

$$
H\left(g r S\left(\mathcal{R}_{-}\right), d^{g r}\right)=S\left(\mathbb{C}[D] \otimes \overline{\mathfrak{g}}^{f}\right) .
$$

Proof. There is a canonical isomorphism $\operatorname{gr} S\left(\mathcal{R}_{-}\right) \simeq S\left(\operatorname{gr} \mathcal{R}_{-}\right)$that induces

$$
H\left(\operatorname{gr} S\left(\mathcal{R}_{-}\right), d^{\mathrm{gr}}\right) \simeq H\left(S\left(\operatorname{gr} \mathcal{R}_{-}\right), d^{\mathrm{gr}}\right) .
$$

By (5.21), we have $H\left(\operatorname{gr} \mathcal{R}_{-}, d^{\mathrm{gr}}\right)=\bigoplus_{p \in \frac{\mathbb{Z}}{2}} H^{p,-p}\left(\operatorname{gr} \mathcal{R}_{-}, d^{\mathrm{gr}}\right)=\mathbb{C}[D] \otimes \overline{\mathfrak{g}}^{f}$ and thus

$$
H\left(\operatorname{gr} S\left(\mathcal{R}_{-}\right), d^{\mathrm{gr}}\right)=\bigoplus_{p \in \frac{\mathbb{Z}}{2}} H^{p,-p}\left(\operatorname{gr} S\left(\mathcal{R}_{-}\right), d^{\mathrm{gr}}\right)=S\left(\mathbb{C}[D] \otimes \overline{\mathfrak{g}}^{f}\right)
$$

where $\overline{\mathfrak{g}}^{f}=\{\bar{a} \in \overline{\mathfrak{g}} \mid[f, a]=0\}$.

Consider another grading $\Delta$ on $S\left(\mathcal{R}_{-}\right)$defined by

$$
\Delta_{J_{\bar{a}}}=\frac{1}{2}-g_{a}, \quad \Delta_{\phi^{\beta}}=g_{\beta}
$$

for $a \in \mathfrak{g}\left(g_{a}\right)$ and $u_{\beta} \in \mathfrak{g}\left(g_{\beta}\right)$. For $\delta \in \frac{\mathbb{N}}{2}$, denote

$$
S\left(\mathcal{R}_{-}\right)[\delta]=\left\{A \in S\left(\mathcal{R}_{-}\right) \mid \Delta_{A}=\delta\right\} .
$$

One can check that (i) $S\left(\mathcal{R}_{-}\right)[\delta]$ is finite dimensional for any $\delta \in \frac{\mathbb{N}}{2}$, (ii) $\left.d_{[0]}\right|_{S\left(\mathcal{R}_{-}\right)[\delta]} \subset S\left(\mathcal{R}_{-}\right)[\delta]$. By Lemma 5.9 and (i) and (ii) in the previous paragraph, $\left(S\left(\mathcal{R}_{-}\right)[\delta], d\right)$ is a good and locally finite complex.

Proposition 5.10. We have

$$
g^{p, q} H\left(S\left(\mathcal{R}_{-}\right)[\delta], d_{[0]}\right) \simeq H^{p, q}\left(g r S\left(\mathcal{R}_{-}\right)[\delta], d^{g r}\right)
$$

for any $p \in \frac{\mathbb{Z}}{2}$. More precisely,

$$
\begin{aligned}
& F^{p} S\left(\mathcal{R}_{-}\right)^{n}[\delta] \cap \operatorname{kerd}_{[0]}=F^{p} S\left(\mathcal{R}_{-}\right)^{n}[\delta] \cap i m d_{[0]} \text { for } n \neq 0, \\
& F^{p} S\left(\mathcal{R}_{-}\right)^{0}[\delta] \cap d_{[0]}^{-1}\left(F^{p+\frac{1}{2}} S\left(\mathcal{R}_{-}\right)^{1}[\delta]\right)=F^{p+\frac{1}{2}} S\left(\mathcal{R}_{-}\right)^{0}[\delta]+F^{p} S\left(\mathcal{R}_{-}\right)^{0}[\delta] \cap \operatorname{ker} d_{[0]} .
\end{aligned}
$$

Proof. See Lemma 4.2 in [5]; the same proof applies.

Corollary 5.11. As differential algebras,

$$
\mathcal{W}_{B R}(\overline{\mathfrak{g}}, f, k)=H^{0}\left(S\left(\mathcal{R}_{-}\right),\left.d_{[0]}\right|_{S\left(\mathcal{R}_{-}\right)}\right) \simeq S\left(\mathbb{C}[D] \otimes \overline{\mathfrak{g}}^{f}\right) .
$$

Proof. This follows from Lemma 5.9 and Proposition 5.10.

The following proposition (Proposition 5.12) is proved by the argument used in Lemma 4.13 in [5].

Proposition 5.12. The differential algebra $\mathcal{W}_{B R}(\overline{\mathfrak{g}}, f)$ is freely generated by

$$
\left\{E_{i}=J_{\bar{u}_{i}}+R_{i} \mid u_{i} \in \mathfrak{g}^{f} \cap \mathfrak{g}\left(g_{i}\right), R_{i} \in F^{g_{i}+\frac{1}{2}} S\left(\mathcal{R}_{-}\right)\left[1 / 2-g_{i}\right]\right\}_{i \in I},
$$

where $\left\{u_{i}\right\}_{i \in I}$ is a basis of $\mathfrak{g}^{f}$. In other words,

$$
\mathcal{W}_{B R}(\overline{\mathfrak{g}}, f) \simeq \mathbb{C}\left[D^{n} E_{i} \mid i \in I, n \in \mathbb{Z}_{\geq 0}\right] .
$$


Proof. In the second equality in (5.25), for any $i \in I, J_{\bar{u}_{i}}$ is an element in $F^{g_{i}} S\left(\mathcal{R}_{-}\right)^{0}\left[1 / 2-g_{i}\right] \cap$ $d_{[0]}^{-1}\left(F^{g_{i}+\frac{1}{2}} S\left(\mathcal{R}_{-}\right)^{1}\right)$. Hence there is an element

$$
E_{i} \in F^{g_{i}} S\left(\mathcal{R}_{-}\right)^{0}\left[1 / 2-g_{i}\right] \cap \operatorname{ker} d_{[0]}
$$

such that $E_{i}=J_{\bar{u}_{i}}+e_{i}$ for some $e_{i} \in F^{g_{i}+\frac{1}{2}} S\left(\mathcal{R}_{-}\right)^{0}\left[1 / 2-g_{i}\right]$. It is enough to show that

$$
\mathcal{W}_{\mathrm{BR}}(\overline{\mathfrak{g}}, f)=S\left(\mathbb{C}[D] \otimes_{\mathbb{C}} \operatorname{Span}_{\mathbb{C}}\left\{E_{i}\right\}\right) .
$$

Since $E_{i} \in \mathcal{W}_{\mathrm{BR}}(\overline{\mathfrak{g}}, f)$, we have $\mathcal{W}_{\mathrm{BR}}(\overline{\mathfrak{g}}, f) \supset S\left(\mathbb{C}[D] \otimes_{\mathbb{C}} \operatorname{Span}_{\mathbb{C}}\left\{E_{i}\right\}\right)$. Conversely, if $A \in$ $\mathcal{W}_{\mathrm{BR}}(\overline{\mathfrak{g}}, f)$, without loss of generality, we can assume that $A \in F^{p} S\left(\mathcal{R}_{-}\right)^{0}[\delta] \cap \operatorname{ker} d_{[0]}$. The corresponding element $[A] \in \operatorname{gr}^{p,-p} S\left(\mathcal{R}_{-}\right)[\delta]$ satisfies $d^{\text {gr }}[A]=0$. Hence, by Lemma [5.9, there is an element $B_{1} \in F^{p} S\left(\mathcal{R}_{-}\right)[\delta] \cap S\left(\mathbb{C}[D] \otimes_{\mathbb{C}} \operatorname{Span}_{\mathbb{C}}\left\{E_{i}\right\}\right)$ such that

$$
A_{1}=A+B_{1} \in F^{p+\frac{1}{2}} S\left(\mathcal{R}_{-}\right)[\delta] \cap \operatorname{ker} d_{[0]} .
$$

Inductively, for $s \in \mathbb{N}$, there is $B_{s} \in F^{p+\frac{s-1}{2}} S\left(\mathcal{R}_{-}\right)[\delta] \cap S\left(\mathbb{C}[D] \otimes_{\mathbb{C}} \operatorname{Span}_{\mathbb{C}}\left\{E_{i}\right\}\right)$ such that

$$
A_{s}=A+B_{1}+\cdots B_{s} \in F^{p+\frac{s}{2}} S\left(\mathcal{R}_{-}\right)[\delta] \cap \operatorname{ker} d_{[0]} .
$$

Since $S\left(\mathcal{R}_{-}\right)[\delta]$ is finite dimensional, $A_{N}=0$ for $N \gg 0$. Hence $A \in S\left(\mathbb{C}[D] \otimes_{\mathbb{C}} \operatorname{Span}_{\mathbb{C}}\left\{E_{i}\right\}\right)$.

Now we can prove the main theorem in this section.

Theorem 5.13. We have

$$
\mathcal{W}(\overline{\mathfrak{g}}, f) \simeq \mathcal{W}_{B R}^{\mathrm{i}}(\overline{\mathfrak{g}}, f)
$$

for the imaginary number $\mathrm{i} \in \mathbb{C}$.

Proof. Recall that $\mathcal{P}(\overline{\mathfrak{g}})$ is the SUSY affine PVA associated with $\mathfrak{g}$ and $\mathcal{I}_{f}$ is the differential algebra ideal of $\mathcal{P}(\overline{\mathfrak{g}})$ generated by $\bar{n}-(f \mid n)$ for $n \in \mathfrak{n}$. Then the image of $\left\{\bar{a}_{\chi} \bar{b}\right\}$ in $\mathcal{P}(\overline{\mathfrak{g}}) / \mathcal{I}_{f}$ is

$$
s(a)\left(\overline{\pi_{\leq 0}[a, b]}+(f \mid[a, b])+k \chi(a \mid b)\right) .
$$

If we denote

$$
j_{\bar{a}}=\mathrm{i}^{a} \bar{a} \text { and } j_{a}=\mathrm{i}^{a} a \text { where } \mathrm{i}^{a}= \begin{cases}\mathrm{i} & \text { if } a \text { is odd }, \\ 1 & \text { if } a \text { is even, }\end{cases}
$$

then, since $j_{\overline{\mathfrak{n}}}=\left\{j_{\bar{n}} \mid n \in \mathfrak{n}\right\}=\overline{\mathfrak{n}}$,

$$
\mathcal{W}(\overline{\mathfrak{g}}, f) \simeq\left(\mathcal{P}(\overline{\mathfrak{g}}) / \mathcal{I}_{f}\right)^{\operatorname{ad}_{\chi} j_{\overline{\mathfrak{n}}}}
$$

Observe the following facts.

(i) Equation (5.26) can be rewritten as

$$
\left\{j_{\bar{a} \chi} j_{\bar{b}}\right\}=s(a, b) s(a)\left(j_{\overline{\pi_{\leq 0}\left[j_{a}, j_{b}\right]}}+\mathrm{i}(f \mid[a, b])\right)+k \chi(a \mid b)
$$

(ii) For $u_{\beta} \in \mathfrak{n}, j_{\bar{\beta}}=j_{\bar{u}_{\beta}}$ and $j_{A}, j_{B} \in \mathcal{P}(\overline{\mathfrak{g}})$, we have

$$
\left\{j_{\bar{\beta} \chi} j_{A} j_{B}\right\}=s(A, \beta) j_{A}\left\{j_{\bar{\beta}} j_{B}\right\}+\left\{j_{\bar{\beta}} j_{A}\right\} j_{B} \in \mathbb{C}[\chi] \otimes \mathcal{P}(\overline{\mathfrak{g}}) .
$$

(iii) For $j_{A} \in \mathcal{P}(\overline{\mathfrak{g}})$,

$$
\left\{j_{\bar{\beta} \chi} D j_{A}\right\}=s(\beta)(D+\chi)\left\{j_{\bar{\beta} \chi} j_{A}\right\} .
$$

On the other hand, associated with $\mathcal{W}_{\mathrm{BR}}^{\mathrm{i}}(\overline{\mathfrak{g}}, f)$, we have the followings: 
(I) For $J_{\bar{a}}$ for $a \in \bigoplus_{i \leq 0} \mathfrak{g}(i)$,

$$
d_{[0]}^{\mathrm{i}}\left(J_{\bar{a}}\right)=\sum_{\beta \in S}\left(s(a, \beta) s(\beta) \phi^{\bar{\beta}} J_{\overline{\left[u_{\beta}, a\right]}}+\mathrm{i} s(a, \beta) s(\beta) \phi^{\bar{\beta}}\left(f \mid\left[u_{\beta}, a\right]\right)-s(\beta) k D \phi^{\bar{\beta}}\left(u_{\beta} \mid a\right)\right) .
$$

(II) For $J_{A}, J_{B}$ in $S\left(\mathbb{C}[D] \otimes J_{\mathfrak{g}_{\leq 0}}\right)$,

$$
d_{[0]}^{\mathrm{i}}\left(J_{A} J_{B}\right)=s(A) J_{A} d_{[0]}^{\mathrm{i}}\left(J_{B}\right)+d_{[0]}^{\mathrm{i}}\left(J_{A}\right) J_{B} .
$$

(III) For $J_{A}$ in $S\left(\mathbb{C}[D] \otimes J_{\mathfrak{g} \leq 0}\right)$,

$$
d_{[0]}^{\mathrm{i}}\left(D J_{A}\right)=-D d_{[0]}^{\mathrm{i}}\left(J_{A}\right) .
$$

Let us consider the differential algebra isomorphism

$$
\phi: \mathcal{P}(\mathfrak{g}) / \mathcal{I}_{f} \rightarrow S\left(\mathbb{C}[D] \otimes J_{\mathfrak{g}_{\leq 0}}\right), \quad j_{\bar{a}} \mapsto J_{\bar{a}} .
$$

Suppose $a \in \mathfrak{g}_{\leq 0}$. Denote the coefficient of $\left(-\chi^{2}\right)^{n_{0}} \chi^{n_{1}}$ for $n_{0} \in \mathbb{Z}_{\geq 0}$ and $n_{1}=0,1$ in $\left\{j_{\bar{\beta}} j_{\bar{a}}\right\}$ by $K_{(\beta, a)}^{n_{0}, n_{1}}$. Then, by (i) and (I), the coefficient of $\partial^{n_{0}} D^{n_{1}} \phi^{\beta}$ in $d_{[0]}^{\mathrm{i}}\left(J_{\bar{a}}\right)$ is $(-s(\beta))^{n_{1}} \phi\left(K_{(\beta, a)}^{n_{0}, n_{1}}\right)$. Moreover, by comparing (ii) and (II) (resp. (iii) and (III)), we have the following statement: For any $j_{A} \in S\left(\mathbb{C}[D] \otimes \mathfrak{g}_{\leq 0}\right)$,

$$
\begin{aligned}
& \left\{j_{\bar{\beta}} j_{A}\right\}=\sum_{n_{0} \in \mathbb{Z}_{\geq 0}, n_{1}=0,1}\left(-\chi^{2}\right)^{n_{0}} \chi^{n_{1}} K_{(\beta, A)}^{n_{0}, n_{1}} \in \mathbb{C}[\chi] \otimes \mathcal{P}(\mathfrak{g}) / \mathcal{I}_{f} \\
& \Longleftrightarrow \quad d_{[0]}\left(\phi\left(j_{A}\right)\right)=\sum_{n_{0} \in \mathbb{Z}_{\geq 0}, n_{1}=0,1}(-s(\beta))^{n_{1}} \partial^{n_{0}} D^{n_{1}} \phi\left(K_{(\beta, A)}^{n_{0}, n_{1}}\right) .
\end{aligned}
$$

Hence we have

$$
\left\{j_{\bar{\beta}} j_{A}\right\}=0 \in \mathbb{C}[\chi] \otimes \mathcal{P}(\mathfrak{g}) / \mathcal{I}_{f} \text { for all } \beta \in S \Longleftrightarrow d_{[0]}^{\mathrm{i}}\left(\phi\left(j_{A}\right)\right)=0 .
$$

Recall that $\mathcal{W}_{\mathrm{BR}}^{\mathrm{i}}(\overline{\mathfrak{g}}, f)=H^{0}\left(S\left(\mathcal{R}_{-}\right), d_{[0]}^{\mathrm{i}}\right)$. Thus

$$
\mathcal{W}_{I}(\overline{\mathfrak{g}}, f)=S\left(\mathbb{C}[D] \otimes J_{\mathfrak{g}_{\leq 0}}\right) \cap \operatorname{ker}\left(d_{[0]}\right)
$$

and $\mathcal{W}_{\mathrm{BR}}^{\mathrm{i}}(\overline{\mathfrak{g}}, f) \simeq \mathcal{W}(\overline{\mathfrak{g}}, f)$ as differential algebras.

Moreover, by comparing (5.19) and (i), we conclude that $\mathcal{W}(\overline{\mathfrak{g}}, f)$ and $\mathcal{W}_{\mathrm{BR}}^{\mathrm{i}}(\overline{\mathfrak{g}}, f)$ are isomorphic as SUSY PVAs.

\section{Structures of SUSY Classical affine W-algebras associated with Lie SUPERALGEBRAS}

In this section, we use the notations from Section 5. Recall that $\mathfrak{g}$ is a simple finite Lie superalgebra with the subalgebra $\operatorname{Span}_{\mathbb{C}}\{E, e, H, f, F\}$ that is isomorphic to osp $(1 \mid 2)$. Using the $\mathfrak{s l}_{2}$ representation theory,

$$
\mathfrak{g}^{F}=\mathfrak{g}^{f} \oplus\left[e, \mathfrak{g}^{f}\right] \quad \text { for } \quad \mathfrak{g}^{f}=\operatorname{ker} \operatorname{ad} f
$$

and, by (3.2), we have

$$
\mathfrak{g}=\mathfrak{g}^{f} \oplus[e, \mathfrak{g}] .
$$

We can take bases

$$
\left\{r_{j} \mid j \in J^{f}\right\} \quad \text { and } \quad\left\{r^{j} \mid j \in J^{f}\right\}
$$

of $\mathfrak{g}^{f}$ and $\mathfrak{g}^{e}$ such that $\left(r^{i} \mid r_{j}\right)=\delta_{i, j}$ and assume that the bases are homogeneous with respect to both parity and the $\mathbb{Z} / 2$-grading on $\mathfrak{g}$. 
Let us denote

$$
r_{m}^{i}=(\operatorname{ad} f)^{m} r^{i} \text { for } m \in \mathbb{Z}_{\geq 0}, i \in J^{f}
$$

and

$$
r_{j}^{n}=C_{j, n}(\operatorname{ad} e)^{n} r_{j} \text { for } C_{j, n} \in \mathbb{C}
$$

such that $\left(r_{m}^{i} \mid r_{j}^{n}\right)=\delta_{m, n} \delta_{i, j}$.

Lemma 6.1. In (6.2), we have

$$
C_{j, n}= \begin{cases}\frac{1}{(m !)^{2}\left(\begin{array}{c}
2 \alpha_{j} \\
m
\end{array}\right)} & \text { if } n=2 m \text { is even }, \\
\frac{-s(j)}{(m+1) ! m !\left(\begin{array}{c}
2 \alpha_{j} \\
m+1
\end{array}\right)} & \text { if } n=2 m+1 \text { is odd. }\end{cases}
$$

Proof. Let us denote $\tilde{r}_{j}^{n}:=(\operatorname{ade})^{n} r_{j}$. Then $\left[f, \tilde{r}_{j}^{n}\right]=\left[-H, \tilde{r}_{j}^{n-1}\right]-\left[e,\left[f, \tilde{r}_{j}^{n-1}\right]\right]$. Hence for $A_{n} \in \mathbb{C}$ such that $\left[f, \tilde{r}_{j}^{n}\right]=A_{n} \tilde{r}_{j}^{n-1}$,

$$
A_{n}=\left(2 \alpha_{j}-n+1\right)-A_{n-1} \text { for } n \geq 2
$$

and $A_{1}=2 \alpha_{j}$. Thus, we obtain $A_{2 m+1}=2 \alpha_{j}-m$ and $A_{2 m}=-m$. Similarly, for $B_{n} \in \mathbb{C}$ such that $\left[e, r_{n}^{j}\right]=B_{n} r_{n-1}^{j}$, we have $B_{2 m+1}=-2 \alpha_{j}+m$ and $B_{2 m}=m$.

Observe that

$$
\begin{aligned}
& \left(r_{n}^{j} \mid \tilde{r}_{j}^{n}\right)=\left(\left[f, r_{n-1}^{j}\right] \mid\left[e, \tilde{r}_{j}^{n-1}\right]\right) \\
& =(-1)^{n-1} s(j)\left(-2 \alpha_{j}+n-1\right)\left(r_{n-1}^{j} \mid \tilde{r}_{j}^{n-1}\right)-\left(\left[e, r_{n-1}^{j}\right] \mid\left[f, \tilde{r}_{j}^{n-1}\right]\right) \\
& =(-1)^{n-1} s(j)\left(-2 \alpha_{j}+n-1\right)\left(r_{n-1}^{j} \mid \tilde{r}_{j}^{n-1}\right)-A_{n-1} B_{n-1}\left(r_{n-2}^{j} \mid \tilde{r}_{j}^{n-2}\right) .
\end{aligned}
$$

We can check $\left(r_{1}^{j} \mid \tilde{r}_{j}^{1}\right)=-s(j) 2 \alpha_{j}$ by direct computations. Moreover, by induction,

$$
\left(r_{n}^{j} \mid \tilde{r}_{j}^{n}\right)= \begin{cases}(m !)^{2}\left(\begin{array}{c}
2 \alpha_{j} \\
m
\end{array}\right) & \text { if } n=2 m \text { is even }, \\
-s(j)(m+1) ! m !\left(\begin{array}{c}
2 \alpha_{j} \\
m+1
\end{array}\right) & \text { if } n=2 m+1 \text { is odd } .\end{cases}
$$

Since $\left(r_{n}^{j} \mid r_{j}^{n}\right)=1$, we get (6.3).

Consider the algebra homomorphisms

$$
\begin{aligned}
& \pi_{S}: \mathcal{P}\left(\overline{\mathfrak{g}}_{\leq 0}\right) \rightarrow \mathcal{P}\left(\overline{\mathfrak{g}}^{f}\right) \\
& \rho_{S}: \mathcal{P}(\overline{\mathfrak{g}}) \rightarrow \mathcal{P}\left(\overline{\mathfrak{g}}_{\leq 0}\right), \quad \bar{a} \mapsto \bar{\pi}_{\leq 0}(\bar{a})+\left(f_{\text {od }} \mid a\right),
\end{aligned}
$$

where $\mathcal{P}(\mathcal{E})=S(\mathbb{C}[D] \otimes \mathcal{E})$ and let

$$
J_{t}^{f}:=\left\{(i, m) \in J^{f} \times \mathbb{Z}_{\geq 0} \mid r_{i}^{m} \in \mathfrak{g}_{t} \text { and } r_{m}^{i} \in \mathfrak{g}_{-t}\right\} .
$$

Lemma 6.2.

(1) If $(i, m) \in J_{h}^{f}$ and $(j, n) \in J_{t}^{f}$ then

$$
\rho_{S}\left\{\bar{r}_{m}^{i} \bar{\gamma}_{j}^{n}\right\}= \begin{cases}0 & \text { if } t-h>\frac{1}{2}, \\ (-1)^{m} s(i) \delta_{i, j} \delta_{n, m+1} & \text { if } t-h=\frac{1}{2}, \\ (-1)^{m} s(i)\left(\overline{\left[r_{m}^{i}, r_{j}^{n}\right]}+\delta_{i, j} \delta_{m, n} k \chi\right) & \text { if } t-h \leq 0,\end{cases}
$$

where $s(i)=s\left(r_{i}\right)$. 
(2) If $\bar{r} \in \mathcal{P}\left(\overline{\mathfrak{g}}^{f}\right)\left(\mathbb{C}[D] \otimes \overline{\left[e, \mathfrak{g}_{\leq-1 / 2}\right]}\right)$ satisfies

$$
\pi_{S} \rho_{S}\left\{\overline{\mathfrak{n}}_{\chi} \bar{r}\right\}=0 \quad \text { for } \quad n \in \mathfrak{g}_{\geq 1 / 2}
$$

then $\bar{r}=0$.

Proof. (1) can be obtained directly from the definition of $\rho_{S}$. To see (2), let us consider

$$
\bar{r}=\sum_{(j, n) \in J_{\leq 0}^{f_{\text {od }}}} F_{j, n}(D) \bar{r}_{j}^{n}
$$

where $F_{j, n}(D)=\sum_{t \in \mathbb{Z}_{\geq 0}} F_{j, n}^{t} D^{t}$ for $F_{j, n}^{t} \in \mathcal{P}\left(\overline{\mathfrak{g}}^{f}\right)$. Suppose $\bar{r} \neq 0$. Then there exists the largest number $k$ such that

$$
(j, n) \in J_{k}^{f}, \quad F_{j, n}(D) \neq 0 .
$$

Then $r_{n-1}^{j} \in \mathfrak{g}\left(-k+\frac{1}{2}\right) \subset \mathfrak{g}_{\geq 1 / 2}$ and

$$
\begin{aligned}
& \pi_{S} \rho_{S}\left\{\bar{r}_{n-1 \chi}^{j} \bar{r}\right\}=\sum_{t \in \mathbb{Z}_{\geq 0}} s\left(F_{j, n}^{t}, r_{n-1}^{j}\right) F_{j, n}^{t}\left\{\bar{r}_{n-1 \chi}^{j} D^{t} \bar{r}_{j}^{t}\right\} \\
& =\sum_{t \in \mathbb{Z}_{\geq 0}} s\left(F_{j, n}^{t}, r_{n-1}^{j}\right) s\left(r_{n-1}^{j}\right)^{t+1} F_{j, n}^{t}(\chi+D)^{t} \neq 0 .
\end{aligned}
$$

Hence, we have proved the lemma.

By Lemma 6.2 and the structure theory of SUSY W-algebras (Proposition 5.12), we have the unique differential algebra isomorphism

$$
\omega_{S}: \mathcal{P}\left(\overline{\mathfrak{g}}^{f}\right) \rightarrow \mathcal{W}(\overline{\mathfrak{g}}, f), \quad \bar{a} \mapsto \omega_{S}(\bar{a})
$$

where $\omega_{S}(\bar{a})=\bar{a}+\gamma_{S}(\bar{a})+\gamma_{\bar{S}}^{\geq 2}(\bar{a})$ for

$$
\gamma_{S}(\bar{a}) \in \mathcal{P}\left(\overline{\mathfrak{g}}^{f}\right) \otimes\left(\mathbb{C}[D] \overline{\left[e_{\mathrm{od}}, \mathfrak{g}_{\leq-1 / 2}\right]}\right), \quad \gamma_{S}^{n}(a) \in \mathcal{P}\left(\overline{\mathfrak{g}}^{f}\right) \otimes\left(\mathbb{C}[D] \overline{\left[e_{\mathrm{od}}, \mathfrak{g}_{\leq-1 / 2}\right]}\right)^{\otimes n}
$$

and $\gamma_{\bar{S}}^{\geq 2}(a)=\sum_{i \geq 2} \gamma_{S}^{i}(a)$.

Theorem 6.3. Let $\pi_{\mathfrak{g}^{f}}: \mathfrak{g} \rightarrow \mathfrak{g}^{f}$ be the projection map and us denote $\bar{g}^{\sharp S}:=\overline{\pi_{\mathfrak{g}^{f}}(g)}$ for $g \in \mathfrak{g}$. For $a \in \mathfrak{g}^{f} \cap \mathfrak{g}(-\alpha)$, we have

$$
\begin{aligned}
\gamma_{S}(\bar{a})=\sum_{p \in \mathbb{Z}_{\geq 0}} & \sum_{\substack{\left(-\alpha-\frac{1}{2}\right) \prec_{S}\left(j_{0}, n_{0}\right) \prec_{S} \cdots \\
\cdots \prec_{S}\left(j_{p}, n_{p}\right) \prec_{S} 0}}\left(\overline{\left[a, r_{n_{0}}^{j_{0}}\right]^{\sharp S}}-\left(a \mid r_{n_{0}}^{j_{0}}\right) k D\right) \\
& {\left.\left[\prod_{t=1, \cdots, p}\left(\overline{\left[r_{j_{t-1}}^{n_{t-1}+1}, r_{n_{t}}^{j_{t}}\right]}\right]^{\sharp S}-\left(r_{j_{t-1}}^{n_{t-1}+1} \mid r_{n_{t}}^{j_{t}}\right) k D\right)\right] \bar{r}_{j_{p}}^{n_{p}+1}, }
\end{aligned}
$$

where

$$
\left(j_{t}, n_{t}\right) \prec_{S}\left(j_{t+1}, n_{t+1}\right) \text { if and only if }\left\{\begin{array}{l}
k_{t+1}-k_{t} \geq \frac{1}{2} \text { where } \\
\left(j_{t}, n_{t}\right) \in J_{k_{t}}^{f} \text { and }\left(j_{t+1}, n_{t+1}\right) \in J_{k_{t+1}}^{f}
\end{array}\right.
$$

and

$$
-\alpha-\frac{1}{2} \prec_{S}\left(j_{0}, n_{0}\right) \quad \text { if and only if } \quad\left(j_{0}, n_{0}\right) \in J_{k_{0}}^{f} \text { for } k_{0} \geq-\alpha \text {. }
$$


Proof. By Lemma 6.2 (2) and

$$
\pi_{S} \rho_{S}\left\{\bar{r}_{m \chi}^{i} \gamma_{\bar{S}}^{\geq 2}(\bar{a})\right\}=0
$$

it is enough to show that $\pi_{S} \rho_{S}\left\{\bar{r}_{m \chi}^{i} \bar{a}+\gamma_{S}(\bar{a})\right\}=0$. Let us write

$$
\begin{aligned}
& \gamma_{S}(\bar{a})[-1]:=\bar{a} \\
& \gamma_{S}(\bar{a})[0]:=\sum_{\left(-\alpha-\frac{1}{2}\right) \prec_{S}\left(j_{0}, n_{0}\right)}\left(\overline{\left[a, r_{n_{0}}^{j_{0}}\right]^{\sharp S}}-\left(a \mid r_{n_{0}}^{j_{0}}\right) k D\right) \bar{r}_{j_{0}}^{n_{0}+1} \\
& \gamma_{S}(\bar{a})[p]:=\sum_{\substack{\left(-\alpha-\frac{1}{2}\right) \prec_{S}\left(j_{0}, n_{0}\right) \prec_{S} \cdots \\
\cdots \prec_{S}\left(j_{p}, n_{p}\right) \prec_{S} 0}}\left(\overline{\left[a, r_{n_{0}}^{j_{0}}\right]^{\sharp_{S}}}-\left(a \mid r_{n_{0}}^{j_{0}}\right) k D\right) \\
& {\left[\prod_{t=1, \cdots, p}\left(\overline{\left[r_{j_{t-1}}^{n_{t-1}+1}, r_{n_{t}}^{j_{t}}\right]^{\sharp S}}-\left(r_{j_{t-1}}^{n_{t-1}+1} \mid r_{n_{t}}^{j_{t}}\right) k D\right)\right] \quad \bar{r}_{j_{p}}^{n_{p}+1}}
\end{aligned}
$$

for $p \geq 1$. Then

$$
\begin{aligned}
\pi_{S} \rho_{S} & \left\{\bar{r}_{m \chi}^{i} \gamma_{S}(\bar{a})[-1]\right\}=-s\left(r_{m}^{i}, a\right) s\left(r_{m}^{i}\right)\left(\overline{\left[a, r_{m}^{i}\right]^{\sharp S}}-k \chi\left(a \mid r_{m}^{i}\right)\right), \\
\pi_{S} \rho_{S}\left\{\bar{r}_{m \chi}^{i} \gamma_{S}(\bar{a})[0]\right\} & \\
= & \sum_{-\alpha-\frac{1}{2} \prec S\left(j_{0}, n_{0}\right)} s\left(r_{m}^{i}, a r_{n_{0}}^{j_{0}}\right) s\left(r_{m}^{i}\right)\left(\overline{\left[a, r_{n_{0}}^{j_{0}}\right]^{\sharp S}}-\left(a \mid r_{n_{0}}^{j_{0}}\right) k(\chi+D)\right) \pi_{S} \rho_{S}\left\{\bar{r}_{m \chi}^{i} \bar{r}_{j_{0}}^{n_{0}+1}\right\} \\
= & \sum_{-\alpha-\frac{1}{2} \prec_{S}\left(j_{0}, n_{0}\right)}-s\left(r_{m}^{i}, a\right) s\left(r_{m}^{i}\right)\left(\overline{\left[a, r_{n_{0}}^{j_{0}}\right]^{\sharp S}}-\left(a \mid r_{n_{0}}^{j_{0}}\right) k(\chi+D)\right)\left(\overline{\left[r_{j_{0}}^{n_{0}+1}, r_{m}^{i}\right]^{\sharp S}}-k \chi\left(r_{j_{0}}^{n_{0}+1} \mid r_{m}^{i}\right)\right) \\
& \quad+s\left(r_{m}^{i}, a\right) s\left(r_{m}^{i}\right)\left(\overline{\left[a, r_{m}^{i}\right]^{\sharp S}}-k \chi\left(a \mid r_{m}^{i}\right)\right) .
\end{aligned}
$$

Hence,

$$
\begin{aligned}
& \pi_{S} \rho_{S}\left\{\bar{r}_{m \chi}^{i} \gamma_{S}(\bar{a})[0]+\gamma_{S}(\bar{a})[1]\right\} \\
= & \sum_{-\alpha-\frac{1}{2} \prec S\left(j_{0}, n_{0}\right)}-s\left(r_{m}^{i}, a\right) s\left(r_{m}^{i}\right)\left(\overline{\left[a, r_{n_{0}}^{j_{0}}\right]^{\sharp S}}-\left(a \mid r_{n_{0}}^{j_{0}}\right) k(\chi+D)\right)\left(\overline{\left(r_{j_{0}}^{n_{0}+1}, r_{m}^{i}\right]^{\sharp S}}-\left(r_{j_{0}}^{n_{0}+1} \mid r_{m}^{i}\right) k \chi\right) .
\end{aligned}
$$

Inductively, one can show that

$$
\pi_{S} \rho_{S}\left\{\bar{r}_{m \chi}^{i} \gamma_{S}(\bar{a})[p]\right\}=-s\left(r_{m}^{i}, a\right) s\left(r_{m}^{i}\right) \Gamma[p]+s\left(r_{m}^{i}, a\right) s\left(r_{m}^{i}\right) \Gamma[p-1],
$$

where

$$
\begin{aligned}
\Gamma[p]= & \sum_{\substack{-\alpha-\frac{1}{2} \prec_{S}\left(j_{0}, n_{0}\right) \prec_{S} \cdots \\
\cdots \prec_{S}\left(j_{p-1}, n_{p-1}\right) \prec_{S}\left(j_{p}, n_{p}\right)}}\left(\overline{\left[a, r_{n_{0}}^{j_{0}}\right]^{\sharp S}}-\left(a \mid r_{n_{0}}^{j_{0}}\right) k(\chi+D)\right) \\
& {\left[\prod_{t=1, \cdots, p}\left(\overline{\left[r_{j_{t-1}}^{n_{t-1}+1}, r_{n_{t}}^{j_{t}}\right]^{\sharp S}}-\left(r_{j_{t-1}}^{n_{t-1}+1} \mid r_{n_{t}}^{j_{t}}\right) k(\chi+D)\right)\right]\left(\overline{\left[r_{j_{p}}^{n_{p}+1}, r_{m}^{i}\right]^{\sharp}}-\left(r_{j_{p}}^{n_{p}+1} \mid r_{m}^{i}\right) k \chi\right) . }
\end{aligned}
$$


Hence,

$$
\pi_{S} \rho_{S}\left\{\bar{r}_{m \chi}^{i} \gamma_{S}(\bar{a})\right\}=\sum_{p \in \mathbb{Z}_{\geq-1}} \pi_{S} \rho_{S}\left\{\bar{r}_{m \chi}^{i} \gamma_{S}(\bar{a})[p]\right\}=0
$$

Lemma 6.4. For $t \in \frac{\mathbb{Z}}{2}$, we have

$$
\sum_{(i, m) \in J_{-t}^{f}} r_{m}^{i} \otimes r_{i}^{m+1}=-\sum_{(j, n) \in J_{t-\frac{1}{2}}^{f}} r_{j}^{n+1} \otimes r_{n}^{j} .
$$

Proof. It suffices to show that for $[f, a] \in \mathfrak{g}_{-t}$ and $[f, b] \in \mathfrak{g}_{t-1 / 2}$,

$$
\sum_{(i, m) \in J_{-t}^{f}}\left([f, a] \mid r_{m}^{i}\right)\left([f, b] \mid r_{i}^{m+1}\right)=-\sum_{(j, n) \in J_{t-\frac{1}{2}}^{f}}\left([f, a] \mid r_{j}^{n+1}\right)\left([f, b] \mid r_{n}^{j}\right) .
$$

Since

$$
\sum_{(i, m) \in J_{-t}^{f}}\left([f, a] \mid r_{m}^{i}\right)\left([f, b] \mid r_{i}^{m+1}\right)=\sum_{(i, m) \in J_{-t}^{f}}-\left(r_{m+1}^{i} \mid a\right)\left([f, b] \mid r_{i}^{m+1}\right)=-([f, b] \mid a)
$$

and

$$
-\sum_{(j, n) \in J_{t-\frac{1}{2}}^{f}}\left([f, a] \mid r_{j}^{n+1}\right)\left([f, b] \mid r_{n}^{j}\right)=\sum_{(j, n) \in J_{t-\frac{1}{2}}^{f}}\left([f, a] \mid r_{j}^{n+1}\right)\left(r_{n+1}^{j} \mid b\right)=([f, a] \mid b),
$$

we have (6.10).

Theorem 6.5. Let $a \in \mathfrak{g}_{-t_{1}}^{f}$ and $b \in \mathfrak{g}_{-t_{2}}^{f}$. Then

$$
\begin{aligned}
& \left\{\omega_{S}(\bar{a})_{\chi} \omega_{S}(\bar{b})\right\}=s(a)(\overline{[a, b]}+\chi k(a \mid b)) \\
& -s(a, b) s(a) \sum_{p \in \mathbb{Z}_{\geq 0}} \sum_{\substack{-t_{2}-\frac{1}{2} \prec_{S}\left(j_{0}, n_{0}\right) \prec_{S} \cdots \\
\cdots \prec_{S}\left(j_{p}, n_{p}\right) \prec_{S} t_{1}}}\left(\omega_{S}\left(\overline{\left[b, r_{m_{0}}^{i_{0}}\right]^{\sharp}}\right)-k\left(b \mid r_{m_{0}}^{i_{0}}\right)(D+\chi)\right) \\
& {\left[\prod_{t=1,2, \cdots, p}\left(\omega_{S}\left(\overline{\left[r_{i_{t-1}}^{m_{t-1}+1}, r_{m_{t}}^{i_{t}}\right]^{\sharp S}}\right)-k\left(r_{i_{t-1}^{m_{t-1}+1}} \mid r_{m_{t}}^{i_{t}}\right)(D+\chi)\right)\right]} \\
& \left.\left(\omega_{S}\left(\overline{\left[r_{i_{p}}^{m_{p}+1}, a\right]}\right]_{S}\right)-k\left(r_{i_{p}}^{m_{p}+1} \mid a\right)(D+\chi)\right) \text {. }
\end{aligned}
$$

Proof. Recall the notations in ([6.9) and, for simplicity, we denote by $\overrightarrow{(i, m)_{0}^{p}}$ the sequence $\left(i_{0}, m_{0}\right), \cdots,\left(i_{p}, m_{p}\right)$ such that

$$
\left(i_{0}, m_{0}\right) \prec_{S}\left(i_{1}, m_{1}\right) \prec_{S} \cdots \prec_{S}\left(i_{p-1}, m_{p-1}\right) \prec_{S}\left(i_{p}, m_{p}\right)
$$

and define $\overrightarrow{(j, n)_{0}^{q}}$ similarly. 
It suffices to show that

$$
\begin{array}{r}
\pi_{S} \rho_{S}\left\{\gamma_{S}(\bar{a})[p]_{\chi} \gamma_{S}(\bar{b})[q]\right\}=-s(a, b) s(a) \sum_{\overline{(i, m)_{0}^{p+q+1}}}\left(\overline{\left[b, r_{m_{0}}^{i_{0}}\right]^{\sharp S}}-k\left(b \mid r_{m_{0}}^{i_{0}}\right)(D+\chi)\right) \\
{\left[\prod _ { t = 1 , 2 , \cdots , p + q + 1 } \left(\overline{\left.\left[r_{\left.i_{t-1}^{m_{t-1}+1}, r_{m_{t}}^{i_{t}}\right]^{\sharp S}}^{\sharp}-k\left(r_{i_{t-1}}^{m_{t-1}+1} \mid r_{m_{s}}^{i_{s}}\right)(D+\chi)\right)\right]}\right.\right.} \\
\left(\overline{\left[r_{\left.i_{p+q+1}^{m_{p+q+1}+1}, a\right]}^{\sharp S}-k\left(r_{i_{p+q+1}}^{m_{p+q+1}+1} \mid a\right)(D+\chi)\right) .}\right.
\end{array}
$$

By the master formula, we have

$$
\begin{gathered}
\left.\pi_{S} \rho_{S}\left\{\gamma_{S}(\bar{a})[p]_{\chi} \gamma_{S}(\bar{b})[q]\right\}=\sum_{-t_{1}-\frac{1}{2} \prec_{S} \overline{(i, m)_{0}^{p}} \prec_{S} 0} s(a, \bar{b}) s\left(a, r_{j_{q}}^{n_{q}}\right)\left(\overline{\left[b, r_{n_{0}}^{j_{o}}\right.}\right]^{\sharp S}-\left(b \mid r_{n_{0}}^{j_{0}}\right)(\chi+D)\right) \\
{\left[\prod_{t=1, \cdots, q}\left(\overline{\left.\left[r_{j_{t-1}}^{n_{t-1}+1} \prec_{S} r_{n_{t}}^{j_{t}}\right]^{\sharp, n}\right)_{0}^{t} \prec_{S} 0}-\left(r_{j_{t-1}}^{n_{t-1}+1} \mid r_{n_{t}}^{j_{t}}\right)(\chi+D)\right)\right] \pi_{S} \rho_{S}\left\{\gamma_{S}(\bar{a})[p]_{\chi} \bar{r}_{j_{q}}^{n_{q}+1}\right\} .}
\end{gathered}
$$

The $\chi$-bracket in the last line of (6.13) can be written as

$$
\begin{aligned}
& \sum_{-t_{1}-\frac{1}{2} \prec_{S} \overrightarrow{(i, m)_{0}^{p}} \prec_{S} 0} \pi_{S} \rho_{S}\left\{\gamma_{S}(\bar{a})[p]_{\chi} \bar{r}_{j_{q}}^{n_{q}+1}\right\} \\
& =s\left(a, r_{i_{0}}^{m_{0}}\right)\left[\prod_{t=1, \cdots, p} s\left(r_{i_{t-1}}^{m_{t-1}+1}, r_{i_{t}}^{m_{t}}\right)\right] s\left(r_{j_{q}}^{n_{q}}, \bar{a} r_{i_{p}}^{m_{p}}\right) \pi_{S} \rho_{S}\left\{\bar{r}_{i_{p}}^{m_{p}+1} \chi+D \bar{r}_{j_{q}}^{n_{q}+1}\right\}_{\rightarrow} \\
& \left.\left[\prod_{t=1, \cdots, p}^{\leftarrow}\left(\overline{\left[r_{i_{t-1}}^{m_{t-1}+1}, r_{m_{t}}^{i_{t}}\right]^{\sharp S}}+\left(r_{i_{t-1}}^{m_{t-1}+1} \mid r_{m_{t}}^{i_{t}}\right) k(D+\chi)\right)\right] \overline{\left(\left[a, r_{m_{0}}^{i_{0}}\right]^{\sharp S}\right.}+\left(a \mid r_{m_{0}}^{i_{0}}\right) k(\chi+D)\right), \\
& =(-1)^{p+2} s\left(r_{j_{q}}^{n_{q}}, a\right)\left(\overline{\left[r_{j_{q}}^{n_{q}+1}, r_{i_{p}}^{m_{p}+1}\right]}-k(\chi+D)\left(r_{j_{q}}^{n_{q}+1} \mid r_{i_{p}}^{m_{p}+1}\right)\right) \\
& \left.\left.\left[\prod_{t=1, \cdots, p}^{\leftarrow}\left(\overline{\left[r_{m_{t}}^{i_{t}}, r_{i_{t-1}}^{m_{t-1}+1}\right]^{\sharp S}}-\left(r_{m_{t}}^{i_{t}}\right) \mid r_{i_{t-1}}^{m_{t-1}+1}\right) k(D+\chi)\right)\right] \overline{\left(\left[r_{m_{0}}^{i_{0}}, a\right]\right.} \sharp^{\sharp S}-\left(r_{m_{0}}^{i_{0}} \mid a\right) k(\chi+D)\right)
\end{aligned}
$$


where $\prod_{t=1, \cdots, p}^{\leftarrow} A_{s}:=A_{p} A_{p-1} \cdots A_{2} A_{1}$. By (6.13)-(6.14)

$$
\begin{aligned}
& \pi_{S} \rho_{S}\left\{\gamma_{S}(\bar{a})[p]_{\chi} \gamma_{S}(\bar{b})[q]\right\}=s(a, b) s(a)(-1)^{p} \sum_{-t_{1}-\frac{1}{2} \prec_{S} \overline{(i, m)_{0}^{p}} \prec_{S} 0} \sum_{-t_{2}-\frac{1}{2} \prec_{S} \overline{(j, n)_{0}^{q}} \prec{ }_{S} 0} \\
& \left(\overline{\left[b, r_{n_{0}}^{j_{0}}\right]^{\sharp_{S}}}-\left(b \mid r_{n_{0}}^{j_{0}}\right)(\chi+D)\right)\left[\prod_{t=1, \cdots, q}\left(\overline{\left[r_{j_{t-1}}^{n_{t-1}+1}, r_{n_{t}}^{j_{t}}\right]^{\sharp_{S}}}-\left(r_{j_{t-1}}^{n_{t-1}+1} \mid r_{n_{t}}^{j_{t}}\right)(\chi+D)\right)\right] \\
& \left(\overline{\left[r_{j_{q}}^{n_{q}+1}, r_{i_{p}}^{m_{p}+1}\right]}-(\chi+D)\left(r_{j_{q}}^{n_{q}+1} \mid r_{i_{p}}^{m_{p}+1}\right)\right) \\
& {\left[\prod_{t^{\prime}=1, \cdots, p}^{\leftarrow}\left(\overline{\left[r_{m_{t^{\prime}}}^{i_{t^{\prime}}}, r_{i_{t^{\prime}-1}}^{m_{t^{\prime}-1}+1}\right]^{\sharp S}}-\left(r_{m_{t^{\prime}}}^{i_{t^{\prime}}} r_{i_{t^{\prime}-1}}^{m_{t^{\prime}-1}+1}\right)(D+\chi)\right)\right]\left(\overline{\left[r_{m_{0}}^{i_{0}}, a\right]^{\sharp S}}-\left(r_{m_{0}}^{i_{0}} \mid a\right)(\chi)\right) .}
\end{aligned}
$$

Now, by Lemma 6.4, we get (6.12). Thus, the theorem follows.

\section{REFERENCES}

[1] P. Bouwknegt, K. Schoutens, W symmetry in conformal field theory. Phys. Rep. 223 (1993), no. 4, 183-276.

[2] B. Bakalov, V. G. Kac, Field algebras, IMRN 3 (2003), 123-159.

[3] A. Barakat, A. De Sole, V. Kac, Poisson vertex algebras in the theory of Hamiltonian equations, Jpn. J. Math. 4 (2009), no. 2, 141-252.

[4] S. Carpentier, U.R. Suh, Supersymmetric Bi-Hamiltonian Systems, arXiv:1911.11843.

[5] A. De Sole, V. G. Kac, Finite vs affine W-algebras, Jpn. J. Math. 1 (2006), 137-261.

[6] A. De Sole, V. G. Kac, D. Valeri, Classical affine W-algebras and the associated integrable Hamiltonian hierarchies for classical Lie algebras, Comm. Math. Phys. 360 (2018), no. 3, 851-918.

[7] A. De Sole, V. G. Kac, D. Valeri, Adler-Gelfand-Dickey approach to classical W-algebras within the theory of Poisson vertex algebras, Int. Math. Res. Not. 21 (2015), 11186-11235.

[8] A. De Sole, V. G. Kac, D. Valeri, Classical W-algebras and generalized Drinfeld-Sokolov bi-Hamiltonian systems within the theory of Poisson vertex algebras, Comm. Math. Phys. 323 (2013), no. 2, 663-711.

[9] A. De Sole, V. G. Kac, D. Valeri, Classical W-algebras and generalized Drinfeld- Sokolov hierarchies for minimal and short nilpotents, Comm. Math. Phys. 331 (2014), no. 2, 623-676. Erratum in Commun. Math. Phys. 333 (2015), no. 3, 1617-1619.

[10] A. De Sole, V. G. Kac, D. Valeri, Classical $W$-algebras for $g l_{N}$ and associated integrable Hamiltonian hierarchies, Comm. Math. Phys. 348 (2016), no.1 265-319.

[11] A. De Sole, V. G. Kac, D. Valeri, Structure of classical (finite and affine) W-algebras, J. Eur. Math. Soc. 18 (2016), no. 9, 1873-1908.

[12] F. Delduc, L. Gallot, Supersymmetric Drinfeld-Sokolov reduction, J. Math. Phys. 39 (1998), no. 9, 4729-4745.

[13] V.G. Drinfel'd, V.V. Sokolov, Lie algebras and equations of Korteseg-de Vries Type. J.Sov.Math. 30 (1984), 1975-2036.

[14] B. Feigin, E. Frenkel, Quantization of the Drinfeld-Sokolov reduction, Phys. Lett. B 246 (1990), no. 1-2, $75-81$.

[15] V. A. Fateev and S. L. Lukyanov, The models of two-dimensional conformal quantum field theory with $\mathrm{Zn}$ symmetry, Internat. J. Modern Phys. A 3 (1988), 507-520.

[16] W. L. Gan, V. Ginzburg, Quantization of Slodowy slices, Int. Math. Res. Not. (2002), 243-255.

[17] R. Heluani, V.G. Kac, Supersymmetric vertex algebras, Commun. Math. Phys. 271 (2007), no. 1, 103-178.

[18] T. Inami, H. Kanno, Generalized N=2 super KdV hierarchies: Lie superalgebraic methods and scalar super Lax formalism. In nite analysis Part A, B (Kyoto, 1991), 419-447, Adv. Ser. Math. Phys. 16, World Sci. Publ., River Edge, NJ, (1992).

[19] T. Inami, H. Kanno Lie Superalgebraic Approach to Super Toda Lattice and Generalized Super KdV Equations Commun. Math. Phys. 136 (1991), 519-542. 
[20] T. Inami, Super-W algebras and generalized super-KdV equations. Strings '90 (College Station, TX, 1990), 321-334, World Sci. Publ., River Edge, NJ, (1991).

[21] T. Inami, H. Kanno, $N=2$ super $K d V$ and super sine-Gordon equations based on Lie super algebra $A(1,1)^{(1)}$, Nuclear Phys. B 359 (1991), no. 1, 201-217.

[22] V. Kac, Vertex algebras for beginners, University Lecture Series, AMS, Vol. 10, 1996 (2nd Ed., AMS, 1998).

[23] V. G. Kac, S.-S. Roan, M. Wakimoto, Quantum reduction for affine superalgebras, Comm. Math. Phys. 241 (2003), 307-342.

[24] V. G. Kac, M. Wakimoto, Quantum reduction and representation theory of superconformal algebras. Adv. Math. 185 (2004), 400-458.

[25] B.A.Kupershmidt, A super Korteweg-de Vries equation: An integrable system Phys. Lett. A 102 (1984), no. 5-6, 213-215.

[26] P. Kulish, A. Zeitlin, Super-KdV equation: classical solutions and quantization, PAMM · Proc. Appl. Math. Mech. 4 (2004), 576-577.

[27] J. Madsen, E. Ragoucy, Quantum Hamiltonian Reduction in Superspace Formalism Nucl. Phys. B429 (1994), $277-290$

[28] A. Molev, E. Ragoucy, U.R. Suh, Supersymmetric W-algebras, arXiv: 1901.06557.

[29] Y.I. Manin, A. O. Radul, A Supersymmetric Extension of the Kadomtsev-Petviashvili Hierarchy, Commun. Math. Phys. 98 (1985), 65-77.

[30] I.N. McArthur On the Integrability of the Super-KdV Equation, Commun. Math. Phys. 148 (1992) , 177-188.

[31] W. Oevel, Z. Popowicz, The Bi-Hamiltonian Structure of Fully Supersymmetric Korteweg-de Vries Systems , Comm. Math. Phys. 139 (1991), no. 3, 441-460.

[32] A. Premet, Special transverse slices and their enveloping algebras, Adv. Math. 170 (2002), 1-55.

[33] U. R. Suh, Structure of classical affine and classical affine fractional W-algebras, J. Math. Phys. 56 (2015) 011706.

[34] U. R. Suh, Structures of classical affine W-algebras associated to Lie superalgebras, J. Math. Phys. 57 (2016) 021703 .

[35] U. R. Suh, Classical Affine W-Superalgebras via Generalized Drinfeld-Sokolov Reductions and Related Integrable Systems, Commun. Math. Phys. 358 (2018), 199-236.

[36] A. B. Zamolodchikov, Infinite extra symmetries in two-dimensional conformal quantum field theory, Teoret. Mat. Fiz. 65 (1985), 347-359.

(U.R. Suh) Department of Mathematical Sciences and Research institute of Mathematics, Seoul

National University, GwanAkRo 1, Gwanak-Gu, Seoul 08826, Korea

E-mail address: uhrisu1@snu.ac.kr 